\title{
Adaptation of Surface Rejuvenation Model to Turbulent Mass Transfer with Coupling Between Thermophoretic and Coulombic Force Interactions
}

\author{
Mingchi Chiou* , Chinghuang Chiu \\ Department of Vehicle Engineering, National Formosa University, Yunlin, Taiwan
}

Email address:

achi@nfu.edu.tw (Mingchi Chiou)

${ }^{*}$ Corresponding author

\section{To cite this article:}

Mingchi Chiou, Chinghuang Chiu. Adaptation of Surface Rejuvenation Model to Turbulent Mass Transfer with Coupling Between Thermophoretic and Coulombic Force Interactions. Engineering Mathematics. Vol. 2, No. 1, 2018, pp. 28-49.

doi: $10.11648 /$ j.engmath.20180201.14

Received: June 7, 2018; Accepted: June 27, 2018; Published: July 24, 2018

\begin{abstract}
Development and applications of the surface rejuvenation model for particle transport towards the surface through turbulent boundary layer involve (1) the introduction of the constitutive equations to establish relations between the fluxes involved in mass transfer processes and the respective driving potentials, (2) the use of definition of the convective velocity to separate the mass transfer flux into diffusive and convective components based on Eulerian models of turbulent deposition proposed by Guha [1] and Young and Leeming [2], (3) the introduction of exponentially distributed functions given by Danckwerts [3] to transform the instantaneous transport properties into the mean domain prior to the solution of the conservation equations, and finally (4) the use of developed relationships for the mean concentration profile and mass transfer flux to obtained an analytical solution for the mean deposition velocity of neutral and charged particles. The deposition velocity of neutral particles with and without including thermophoretic effect is first calculated and then used to clarify the effects caused by the presence of external imposed electric field. A comparison with the experimental data and other theoretical predictions shows that the surface rejuvenation model is logical in finding the combined effect of thermophoresis and turbophoresis on the deposition of neutral particles and confirming the relative independence of deposition velocity upon these drift mechanisms at high values of particle inertia. When both thermophoretic and Coulombic forces operate together, two important conclusions are obtained from this model: (1) the contribution to particle deposition velocities does not represent the sum of these drift mechanisms considered in isolation; (2) the turbophoresis induces an additional drift velocity toward the wall in the vicinity of the wall, alleviating these external forces and enhancing particle deposition onto the wall through reduced build-up of particle concentration adjacent to the wall.
\end{abstract}

Keywords: Brownian Diffusion, Turbulent Diffusivity of Particles, Turbophoresis, Thermophoresis, Coulombic Force, External Imposed Electric Field

\section{Introduction}

Transport and deposition of immersed particles from turbulent flowing suspensions to adjoining surface have received considerable attention in industrial and environmental applications, examples being the chemical spray coating of metals, the contamination of semiconductor elements during manufacture, and the fouling of heat exchange equipments. An essential feature lies in the ability to predict the deposition rate of immersed particles for quantitative understanding of the dominant transport mechanisms.
Montgomery and Corn [4] reviewed the free-flight models [5-7] and consequently tabled the measured deposition velocities of micron-sized aerosol particles $(0.44-2.16 \mu \mathrm{m})$ for evaluating the theoretical predictions. It should be mentioned that Friedlander and Johnstone [5] were first to offer a theoretical calculation method for observing large increase in deposition rates, and that the fundamental difference between different models of this type lies in 
prescribing the initial velocity $v_{i}$ that the particles possess at the distance where they effectively breaks away from the containing eddies and embarks on a free flight towards the wall. In Davies's calculations [6], the initial velocity $\left(v_{i}^{+}=v_{i} / u_{*}\right)$ was assumed to be the same as the local rms fluctuation velocity $\left.\overline{\left(v_{f}^{+2}\right.}=\overline{v_{f}^{\prime 2}} / u_{*}^{2}\right)$ of local fluid, but the calculations for deposition velocity obtained on the basis of this assumption were lower by some two orders of magnitude than the measured values. In order to improve this discrepancy, Liu and Illori [8] proposed a new expression for particle eddy diffusivity $\varepsilon_{p}$,

$$
\varepsilon_{p} / v=\varepsilon_{t} / v+\tau_{p}^{+} \overline{v_{f}^{\prime+2}}
$$

where the turbulence induced eddy viscosity is designated by $\varepsilon_{t}, v$ and $u_{*}$ are the fluid kinematic viscosity and friction velocity, respectively. The dimensionless particle relaxation time $\tau_{p}^{+}\left(=\tau_{p} u_{*}^{2} / v\right)$ is a measure of the particle inertia relative to the viscous drag resistance that a particle experiences, and can also be interpreted as an inertial time constant required to adjust a different fluid fluctuation velocity surrounding the particle. Hence, $\tau_{p}^{+} \overline{v_{f}^{\prime 2}}$ acts as an additional term to the commonly used $\varepsilon_{p}=\varepsilon_{t}$ account for enhanced deposition by inertia at larger values of $\tau_{p}^{+}$.

For particles subject to aerodynamic resistance in the Stokes drag regime, this inertial time constant with the Cunningham correction $C_{c}=1+\left[15.6+7.0 \exp \left(-0.059 P d_{p}\right)\right] / P d_{p}$ given by Hinds [9] is defined as

$$
\tau_{p}^{+}=\frac{2}{9} C_{c} \frac{\rho_{p}}{\rho_{f}} r_{p}^{+2},
$$

where $P$ is the absolute pressure in $k P a, d_{p}$ the particle diameter in $\mu \mathrm{m}, v$ the kinematic viscosity of carrier fluid, $\rho_{p} / \rho_{f}$ the ratio of particle density $\rho_{p}$ to fluid density $\rho_{f}, r_{p}^{+}=r_{p} u_{*} / v$ the normalized particle radius $r_{p}$, and $u_{*}$ the friction velocity. The measured deposition velocities of olive oil particles $(1.4-21.0 \mu \mathrm{m})$ on glass and copper pipe surfaces from Liu and Agarwal [10] are generally accepted as one of the most dependable data set for comparison with the theoretical predictions, where the dependences of calculated deposition velocities $v_{d}^{+}$on the particle size are presented as a function of particle relaxation time $\tau_{p}^{+}=u_{*}^{2} \tau_{p} / \nu$. Experimental data in term of $v_{d}^{+}$versus $\tau_{p}^{+}$are usually divided into three regimes: the diffusional-deposition regime, the particle eddy diffusion-impaction regime and the particle inertia moderated regime. In the diffusional-deposition regime, particles faithfully follow the turbulent fluctuations of fluid motion, the damping of the turbulent intensities by viscous effects causes them to get trapped in a thin viscous sublayer, where particle transport towards the wall is associated with Brownian motion and $v_{d}^{+}$decreases monotonically with increasing $\tau_{p}^{+}$. In the particle eddy diffusion-impaction regime, particles acquire an impacting momentum towards the wall induced by the turbulent eddies in the outer boundary layer and then coast across the viscous sublayer to the wall as a result of their inertia, and the trend in $v_{d}^{+}$rises steeply by several orders of magnitude as $\tau_{p}^{+}$increases. In the particle inertia moderated regime, the response to the turbulent fluctuations is slow and the fluctuating velocities of fluid seen by particles become progressively larger with increasing particle inertia, which is responsible for a scanty decline in $v_{d}^{+}$from its maximum value beyond certain $\tau_{p}^{+}$.

The trend of experimental results provides evidence that the enhanced factor $\overline{v_{f}^{\prime 2}} \tau_{p}^{+}$certainly plays a key role in the inertial deposition mechanism, but the change of $\varepsilon_{p} / v$ with $\overline{v_{f}^{+2}} \tau_{p}^{+}$is not monotonic because of the existence of particle inertia moderated regime which is characterized by slightly decrease in $v_{d}^{+}$with increase in $\tau_{p}^{+}$. Kallio and Reeks [11] successfully reproduced $v_{d}^{+}$of inertia-dominated particles in terms of the predicted trends, by using the Lagrangian particle tracking technique in a numerically generated two-dimensional turbulent channel flow. They also demonstrated that the increase in $\overline{v_{f}^{\prime 2}} \tau_{p}^{+}$with $\tau_{p}^{+}$cannot be simply manifested as an increased diffusivity in the wall boundary layer. Eulerian models of turbulent deposition have provided a method for handling the particle mass and momentum conservation equations, which emphasize the different roles played by respective driving potentials in the mean mass transfer flux to be decomposed into diffusive and convective components [1, 2]. The analytical procedure of this approach was useful in stressing the importance of turbophoresis on the convective flux of particles with substantial inertia in turbulent boundary layers and confirming the relative independence of $v_{d}^{+}$on $\tau_{p}^{+}$at large values.

The important role that the wall-normal velocity component, fluctuating between local regions near the wall, plays in the inertial deposition mechanism, was also an important result for turbulent transport of a transferable quantity close to the wall observed in the visual flow studies $[12,13]$. They too found that although most fluid eddies penetrate the wall region to varying depths close to the wall, only occasional eddies actually accelerate and displace the low-momentum fluid at which they are arriving. This results in the establishment of a periodically developing boundary layer above an unreplenished layer of fluid, which has been reported to remain adjacent to the wall. Since the concentration gradient of immersed particles will be momentarily steeper near the fresh fluid, Harriott [14] modified the surface rejuvenation 
model proposed by Danckwert [3] by assuming that the fluid eddies arriving at various times come to within various small distances of the surface and that, presumably during the residency of an individual eddy within the close vicinity of the interface, unsteady molecular transport is presumed to control immersed particles toward the wall. Bullin and Dukler [15] successfully simplified Harriott's calculation procedure, by a stochastic computational concept to calculate the mean mass transfer coefficient in terms of unspecified parameters of the mean eddy approach distance and the mean residence time between two successive eddies. The viscous sublayer influx concept of a wallward flow induced fluid renewal within the viscous sublayer was introduced by Brocker [16], which leads to an analytical solution for the turbulent transport properties with the aid of the surface rejuvenation model. Also, the physically reasonable presumptions of unsteady momentum, heat and mass transfer to govern the area within the wall region for the period of time between two successive eddies have been described in detail. The variable of principal interest is the mean residence time $\bar{\tau}^{+}\left(=u_{*} \sqrt{\bar{\tau} / v}\right)$ between two successive eddies. Although the result $\bar{\tau}^{+}=18$ for Reynolds number $\operatorname{Re} \geq 10^{4}$ was found in the experimental data [17-19], this model provides a basis for the development of relationship for $\bar{\tau}^{+}$.

With the consideration of the potential usefulness of practical calculations for understanding the particle transport mechanisms in the form of analytical equations, the main objective of this work is similar to that of Guha [1], but the formulating solution of the surface rejuvenation model developed by Brocker [16] will be adapted to momentum, heat and mass transfer, with emphasis given to predictions for the mean deposition velocity of charged particles that carry the saturation charge in the presence of external electric fields. Based on the assumption that when an external filed is present the direction of the field is toward the wall, the momentum equation of particles includes only the Coulomb force in addition to the dominant deposition mechanisms. The effects of Coulomb force on the variations of $v_{d}^{+}$with $\tau_{p}^{+}$will be accounted for the varying charge levels based on the number of charges acquired by diffusion, field and combined charging mechanisms of particles at the saturation charge level. In order to clarify the deposition characteristics in the presence of imposed electrical fields, the predicted deposition velocities obtained by this formulating method for neutral particles are also plotted in the figure together with the experimental data $[4,10]$, the numerical results [11] and free-flight model predictions [5-7]. The basic formulation of the surface rejuvenation model will first be presented, including a brief review of the approach developed by Sonke Brocker [16]. This will provide the analytical relationships for the mean residence time $\bar{\tau}^{+}$and the mean thermophoretic velocity $\bar{V}_{t h}^{+}\left(=\bar{V}_{t h} / u_{*}\right)$ in terms of the mean wall shear stress and the mean heat transfer flux, respectively.

\section{Review of the Surface Rejuvenation Model}

The mathematical formulation of the surface rejuvenation model is based on the assumptions that fluid eddies intermittently move from turbulent core to within various small distances $H$ of the surface and that, presumably during the residency $\tau$ of an individual fluid eddy near the wall, the unsteady transport processes of momentum and energy can be described by equations of the form

$$
\begin{aligned}
& \qquad \frac{\partial \varphi}{\partial t}=\Re \frac{\partial^{2} \varphi}{\partial y^{2}}, \\
& \varphi=\varphi_{\infty}[U(y-H)]+g(y)[1-U(y-H)] \text { at } t=0, \\
& \varphi=\varphi_{w} \quad \text { at } y=0, \\
& \varphi=\varphi_{\infty} \quad \text { at } y \rightarrow \infty,
\end{aligned}
$$

where $y$ is the normal distance from the wall and $t$ is the instantaneous contact time starting from the last instant of eddy-rejuvenation cycle. The multiple variable $\varphi(y, t)$ denotes the mean axial velocity $U_{f}(y, t)$ in the case of momentum transfer and the mean temperature $T(y, t)$ in the case of heat transfer. Since the turbulent flux terms $\overline{u_{f}^{\prime} v_{f}^{\prime}}$ and $\overline{T^{\prime} v_{f}^{\prime}}$ are due to the same mechanism of the mean convection, so that their ration of a dimensionless ratio of the turbulent Prandtl number $\operatorname{Pr}_{t}=\varepsilon_{t} / \alpha_{t}$ ought to be of order unity, it is assumed that the transfer coefficient $\mathfrak{R}$ is referred to the enhanced kinematic viscosity $v+\varepsilon_{t}$ and thermal diffusivity $\alpha+\varepsilon_{t}$ as a result of the turbulence induced eddy viscosity $\varepsilon_{t}$. Furthermore, the initial and boundary conditions are given for a specified wall value $\varphi_{w}$ and the initial profile $g(y)$ at first instant of rejuvenation for $y<H$. Since eddies arise from the turbulent core region, $\varphi_{\infty}$ is the velocity or temperature at the first instant of rejuvenation and may be taken as the mean buck values.

The key aspects of formulating solution of the surface rejuvenation model proposed by Brocker [16] lie in (1) the introduction of exponentially distributed functions $p_{t}(t)$, $p_{H}(H)$ and $p_{g}(g)$ into differential formulation in accordance with the residence time distribution $p_{\tau}(\tau)=\exp (-\tau / \bar{\tau}) / \bar{\tau}$ given by Danckwerts [3], (2) the definition of the mean profile

$$
\bar{\varphi}=\int_{0}^{\infty} p_{t}(t) d t \int_{0}^{\infty} p_{H}(H) d H \int_{0}^{\infty} p_{g}(g) d g,
$$

which transforms the differential equation and boundary conditions into the mean domain, (3) the use of $\bar{g}(y)=\bar{\varphi}(y)$ to obtain a system of equations for mean transport based on the distribution functions $p_{t}(t)$ and $p_{\tau}(\tau)$ that are identical. Finally, an approach to the solution of these 
modelling equations leads to an expression for the spatial mean profile. This relationship takes the form

$$
\frac{\bar{\varphi}-\varphi_{\infty}}{\varphi_{w}-\varphi_{\infty}}=\frac{J_{2 \bar{H} / \sqrt{\Re \bar{\tau}}}[(2 \bar{H} / \sqrt{\Re \bar{\tau}}) \exp (-y / 2 \bar{H})]}{J_{2 \bar{H} / \sqrt{\Re} \bar{\tau}}[2 \bar{H} / \sqrt{\Re \bar{\tau}}]}
$$

Furthermore, with the consideration of boundary conditions

$$
\begin{aligned}
& \bar{U}_{f}=u_{\infty} \text { and } \bar{T}=T_{\infty} \text { as } \quad y \rightarrow \infty, \\
& \bar{U}_{f}=0 \quad \text { and } \quad \bar{T}=T_{w} \quad \text { at } \quad y=0,
\end{aligned}
$$

the proposed relationships for the spatial mean velocity and temperature profile can be yielded

$$
\begin{gathered}
\frac{\bar{U}_{f}-u_{\infty}}{-u_{\infty}}=\frac{J_{2 \omega}[2 \omega \exp (-y / 2 \bar{H})]}{J_{2 \omega}[2 \omega]}, \\
\frac{\bar{T}-T_{\infty}}{T_{w}-T_{\infty}}=\frac{J_{2 \vartheta}[2 \vartheta \exp (-y / 2 \bar{H})]}{J_{2 \vartheta}[2 \vartheta]},
\end{gathered}
$$

where the transport parameters $\omega=\bar{H} / \sqrt{\left(v+\varepsilon_{t}\right) \bar{\tau}}$ and $\vartheta=\bar{H} / \sqrt{\left(\alpha+\varepsilon_{t}\right) \bar{\tau}}$ act as a dominating parameter for characterizing the radial inertia of turbulent fluctuation fractions intermittently moving from the turbulent core to near the wall.

\subsection{Formulation for Mean Mass Transfer Properties}

Adaptation of the surface rejuvenation model to the deposition of particles from turbulent flowing suspensions to adjoining the surface is restricted to spherical and dilute suspensions, meaning that the fluid motion is unaffected by the presence of particles and that the collision between particles can be neglected. In analogy with the supplementary flux of momentum and heat evolved by velocity fluctuations, the effect on the transport of immersed particles by turbulence vanishing toward the wall is separated from other effects by decomposing the instantaneous velocity $v_{p}=V_{p}+v_{p}^{\prime}$ and concentration $c=C+c^{\prime}$ into their respective mean components $V_{p}$ and $C$, and fluctuating components $v_{p}^{\prime}$ and $c^{\prime}$. For any given eddy-rejuvenation cycle, the time dependent equations of continuity and balance of momentum for particle transport with both the mean and the fluctuating quantities might be approximate by

$$
\begin{gathered}
\frac{\partial C}{\partial t}+\frac{\partial}{\partial y}\left(C V_{p}+\overline{c^{\prime} v_{p}^{\prime}}\right)=0 . \\
\frac{\partial V_{p}}{\partial t}=-\frac{\partial \overline{v_{p}^{\prime 2}}}{\partial y}-\frac{V_{p}}{\tau_{p}}-\frac{D_{B}}{\tau_{p} C} \frac{\partial C}{\partial y}+\frac{V_{t h}}{\tau_{p}}+\frac{V_{e}}{\tau_{p}}
\end{gathered}
$$

where $y$ is wall-normal distance, $V_{t h}$ and $V_{e}$ are the mean thermophoretic and electrical drift velocity, respectively. As proposed by Hinds [9], the Brownian diffusion $D_{B}$ for a rarefied gas effect can be calculated by $D_{B}=C_{c} \frac{K_{b} T}{3 \pi \mu d_{p}}$, where $T$ is the absolute temperature, $\mu$ the dynamical viscosity and Boltzmann's constant $K_{b}=1.38 \times 10^{-23} \mathrm{~J} / \mathrm{K}$. The mass transfer flux $N$ in the wall-normal direction is given by

$$
N=C V_{p}+\overline{c^{\prime} v_{p}^{\prime}}
$$

Since the existence of the correlation $\overline{c^{\prime} v_{p}^{\prime}}$ arises from the same mechanism of time-mean fluid convection, the mass transfer flux due to turbulent fluctuation is modeled by the gradient diffusion, $\overline{c^{\prime} v_{p}^{\prime}}=-\varepsilon_{p} \partial C / \partial y$, with the proportionality constant of particle eddy diffusivity $\varepsilon_{p}$.

The adaptation of the surface rejuvenation model to the mass transfer is based on the assumption that the comparable mechanisms which dominate the particle transport processes have been evaluated prior to the solution of concentration boundary development. This can be reached by separating the absolute particle velocity $V_{p}$ into the mean diffusive and convective components with the aid of the convective velocity $V_{p c}$ of particles [1],

$$
V_{p}=V_{p c}-D_{B} \partial \ln C / \partial y+V_{t h}+V_{e} .
$$

This has the effect of shifting the concentration dependent terms from equation (11) into equation (10) and leads to the relation

$$
\frac{\partial C}{\partial t}=\frac{\partial}{\partial y}\left[\left(D_{B}+\varepsilon_{p}\right) \frac{\partial C}{\partial y}-V_{t h} C\right]-\frac{\partial}{\partial y}\left[\left(V_{p c}+V_{e}\right) C\right] .
$$

The mass transfer flux which consists of a diffusive and a convective flux toward the wall through the turbulent boundary layer, can then be defined as

$$
N=-\left[\left(D_{B}+\varepsilon_{p}\right) \partial C / \partial y-C V_{t h}\right]+C V_{p c}+C V_{e} .
$$

Consequently, the change of mean particle convection velocity during the residence time $\tau$ between two successive eddies is reduced to a balance between the viscous drag resistance and the turbophoretic drift effect, and can be solved independently from equation as the form

$$
\frac{d V_{p c}}{d t}+\frac{V_{p c}}{\tau_{p}}=-\frac{d \overline{v_{p}^{\prime 2}}}{d y} .
$$

Since the momentum of turbulent fluid element is usually transported much more rapidly than the suspended particles, the turbulent intensity gradient of the uniform diffusion substance could be negligibly small. Hence, at the time just after the arrival of the turbulent fluid elements, the mean distribution of diffusing substance in a turbulent element approaching the wall may subject to the initial and boundary conditions, 


$$
\begin{aligned}
& V_{p c}=f(y)\left[1-U(y-H) \text { and } C=c_{\infty}\left[U\left(y-H_{c}\right)\right]+h(y)\left[1-U\left(y-H_{c}\right)\right] \text { at } \tau=0,\right. \\
& C=c_{\infty} \text { as } y \rightarrow \infty \\
& C=c_{w} \text { at } y=r_{p} .
\end{aligned}
$$

$f(y)$ is the initial profile of the mean particle convection velocity in the uninfluenced layer between the wall and the rejuvenation distance $H$, which highlights the gradient in particle fluctuating velocity that arises primarily due to the sharp decay in fluctuating intensity of surrounding fluid as the wall is approached. The initial profile $h(y)$ of mean particle concentration is considered to be ranged between the wall and the thickness of particle concentration boundary layer $H_{c}$. This characteristic thickness $H_{c}$ is taken into account for the fact that thickness of the concentration boundary layer at the interface is very thin indeed, and also can be considered as a parameter that characterizes the statistical behaviour of particles at take off distance originating from a point source in homogeneous turbulent field.

It has been demonstrated that the form of the distribution density functions $p_{\tau}(\tau)$ of the residence time $\tau$ over the surface do not appear greatly to affect calculated profile of the surface approach models $[18,19]$. Therefore, the analogical forms of $p_{H_{c}}\left(H_{c}\right)=\exp \left(-H_{c} / \bar{H}_{c}\right) / \bar{H}_{c}$ and $p_{h}(h)=\exp (-h / \bar{h}) / \bar{h}$ are assumed for the mean approach distance $H_{c}$ and the initial profile $h(y)$ of mean particle concentration, and $\quad p_{H}(H)=\exp (-H / \bar{H}) / \bar{H} \quad$ and $p_{f}(f)=\exp (-f / \bar{f}) / \bar{f}$ for the mean approach distance $H$ and the initial profile $f(y)$ of mean particle convective velocity. Also, since the quantities considered in the conservation equations are statistical in nature with corresponding distribution density functions, the transformation of mean transport properties into the average domain period is done by defining the average profiles by the equations

$$
\begin{aligned}
& \bar{C}=\int_{0}^{\infty} p_{\tau}(\tau) d \tau \int_{0}^{\infty} p_{H_{c}}\left(H_{c}\right) d H_{c} \int_{0}^{\infty} p_{h}(h) C d h, \\
& \bar{V}_{p c}=\int_{0}^{\infty} p_{\tau}(\tau) d \tau \int_{0}^{\infty} p_{H}(H) d H \int_{0}^{\infty} p_{f}(f) V_{p c} d f .
\end{aligned}
$$

Starting this transformation by multiplying each term of equations (14) and (16) with $p_{\tau}(\tau) d \tau$ and by integrating yields

$$
\begin{gathered}
\tilde{C}-h(y)\left[1-U\left(y-H_{C}\right)\right]-c_{\infty} U\left(y-H_{C}\right)=D_{p} \bar{\tau} \frac{d^{2} \tilde{C}}{d y^{2}}-v_{r} \bar{\tau} \frac{d \tilde{C}}{d y} \\
\tilde{V}_{p c}-\frac{\tau_{p}}{\bar{\tau}+\tau_{p}} f(y)[1-U(y-H)]=-\frac{\bar{\tau} \tau_{p}}{\bar{\tau}+\tau_{p}} \frac{d \overline{\overline{v_{p}^{\prime 2}}}}{d y}
\end{gathered}
$$

where $\tilde{C}=\int_{0}^{\infty} C \exp (-\tau / \bar{\tau}) / \bar{\tau} d \tau, \quad \tilde{V}_{p c}=\int_{0}^{\infty} V_{p c} \exp (-\tau / \bar{\tau}) / \bar{\tau} d \tau$, and $\overline{\overline{v_{p}^{\prime 2}}}=\int_{0}^{\infty} \overline{v_{p}^{\prime 2}} \exp (-\tau / \bar{\tau}) / \bar{\tau} d \tau$. The recomposed particle diffusivity $D_{p}=D_{B}+\varepsilon_{p}$ indicates a comparable effect between the molecular diffusion and the turbulent diffusivity of particles, and the inertial and external properties are manifested in the form of the average drift velocity $\bar{v}_{r}=\bar{V}_{p c}+\bar{V}_{t h}+\bar{V}_{e}$. Next, multiplication of equations (19) and (20) with $p_{h}(h) p_{H_{c}}\left(H_{c}\right) d h d H_{c}$ and $p_{f}(f) p_{H}(H) d f d H$, respectively, and integration with respect to the corresponding variables gives

$$
\begin{gathered}
\bar{C}-\bar{h}(y) e^{\frac{-y}{\bar{H}_{C}}}-c_{\infty}\left(1-e^{\frac{-y}{\bar{H}_{C}}}\right)=D_{p} \bar{\tau} \frac{d^{2} \bar{C}}{d y^{2}}-\bar{\tau} \bar{v}_{r} \frac{d \bar{C}}{d y}, \\
\bar{V}_{p c}-\frac{\tau_{p}}{\bar{\tau}+\tau_{p}} \bar{f}(y) e^{\frac{-y}{\overline{\bar{H}}}}=-\frac{\bar{\tau} \tau_{p}}{\bar{\tau}+\tau_{p}} \frac{\overline{\overline{v_{p}^{\prime 2}}}}{d y} .
\end{gathered}
$$

Because the use of Danckwert's random contact time distribution and residence time distribution results in $\bar{f}(y)=\bar{V}_{p c}$ and $\bar{h}(y)=\bar{C}(y)$, which reveal that the average profiles at the end of the time intervals are identical to the time average profiles that are averaged out over the time intervals, Equation (21) can be reduced to

$$
\frac{d^{2} \bar{C}}{d y^{2}}-\frac{\bar{\tau}_{r}}{D_{p} \bar{\tau}} \frac{d \bar{C}}{d y}=\frac{\bar{C}-c_{\infty}}{D_{p} \bar{\tau}}\left[1-\exp \left(-y / \bar{H}_{c}\right)\right]
$$

with boundary conditions

$$
\bar{C}=c_{w} \text { at } y=r_{p} \text { and } \bar{C}=c_{\infty} \text { as } y \rightarrow \infty .
$$

Also, a constitutive relation for the convective velocity of particles is derived and expressed as

$$
\bar{V}_{p c}-\frac{\tau_{p}}{\bar{\tau}+\tau_{p}} \bar{V}_{p c} \exp (-y / \bar{H})=-\frac{\bar{\tau} \tau_{p}}{\bar{\tau}+\tau_{p}} \frac{d \overline{\overline{v_{p}^{\prime 2}}}}{d y} .
$$

Based on the consideration that the fluid velocity fluctuations seen by suspended particles should increase with increasing particle diameter, Mito and Hanratty [20] proposed that the location of about one particle radius from the wall can be taken into account for the fluctuating influences by fluid turbulence. Accordingly, during the average sublayer growth period $\bar{\tau}$, the convective velocity of particles induced by the wall-normal gradient of turbulent fluctuation intensity can be approximately evaluated by 


$$
\bar{V}_{p c}^{+}=\frac{\bar{\tau}^{+}}{\bar{\tau}^{+}+\tau_{p}^{+}\left[1-\exp \left(\frac{-r_{p}^{+}}{\bar{H}^{+}}\right)\right]}\left(-\left.\tau_{p}^{+} \frac{\overline{\overline{v_{p}^{\prime 2}}}}{d y^{+}}\right|_{y^{+}=r_{p}^{+}}\right)
$$

where $\quad \bar{V}_{p c}^{+}=V_{p c} / u_{*}, \quad \overline{\overline{v_{p}^{\prime 2}}}=\overline{\overline{v_{p}^{\prime 2}}} / u_{*}^{2}, \quad \bar{\tau}^{+}=u_{*} \sqrt{\bar{\tau} / v}$, $y^{+}=y u_{*} / v$ and $H^{+}=H u_{*} / v$. This highlights the fact that turbophoresis emerges in both the gradient of particle fluctuating velocity and the inertia of particles, and enhances the convective flux toward the wall through the induced convective drift velocity against the sharp decay in the fluctuating intensity of surrounding fluid. Since the time mean vales of the fluid and particle turbulence flux arise from the same mechanism of mean fluid convection, a linear differential equation for Reynolds normal stress of the particles in the transverse direction proposed by Shin et al. [21] may reasonably be modified by substituting the residence time $\tau$ of an individual eddy element for the instantaneous contact time, and written as

$$
\frac{d v_{p}^{\prime}}{d \tau}+\frac{1}{\tau_{p}} v_{p}^{\prime}=\frac{1}{\tau_{p}} v_{f}^{\prime}
$$

From the Lagrangian correlation coefficient $\Re_{L f}(\tau)=\exp \left(-\tau / \tau_{g}\right)=\overline{v_{f}^{\prime}(0) v_{f}^{\prime}(\tau)} / \overline{v_{f}^{\prime 2}}$ of the fluid motion in the stationary homogeneous uniform flow, the solution, which satisfies the initial condition $v_{p}^{\prime}=0$ at $\tau=0$, for the radial fluctuation velocity of particles can be expressed as

$$
\overline{v_{p}^{\prime 2}}(\tau)=\overline{v_{f}^{\prime 2}}(\tau)-2 \overline{v_{f}^{\prime}(\tau) v_{f}^{\prime}(0)} e^{-\frac{\tau}{\tau_{p}}}+\overline{v_{f}^{\prime 2}}(0) e^{-\frac{2 \tau}{\tau_{p}}}=\overline{v_{f}^{\prime 2}}\left[1-2 e^{-\left(\frac{\tau_{g}+\tau_{p}}{\tau_{g} \tau_{p}}\right) \tau}+e^{-\left(\frac{2}{\tau_{p}}\right) \tau}\right],
$$

where $\tau_{g}=\frac{\varepsilon_{t}}{\overline{v_{f}^{2}}}$ is integral time scale of the Lagrangian correlation. Simultaneously, the radial particle fluctuation velocity of a single eddy can also be transformed into its average time domain of all eddy lifetimes and thus, during the average growth period of developing sublayer, a constitutive relation for the average fluctuation velocities of immersed particles and carrier fluid can be expressed as

$$
\overline{\overline{{v_{p}^{\prime+2}}^{\prime 2}}}=\overline{{v_{f}^{\prime 2}}^{\prime 2}}\left(1-\frac{2 \tau_{g}^{+} \tau_{p}^{+}}{\bar{\tau}^{+2} \tau_{g}^{+}+\bar{\tau}^{+2} \tau_{p}^{+}+\tau_{g}^{+} \tau_{p}^{+}}+\frac{\tau_{p}^{+}}{2 \bar{\tau}^{+2}+\tau_{p}^{+}}\right)
$$

Under the large density ratio between particle and fluid, the integral time scale of the Lagrangian correlation $\tau_{g}^{+}=u_{*}^{2} \tau_{g} / v=\left(\varepsilon_{t} / v\right) / \overline{v_{f}^{+2}}$ was given by Young and Leeming [2]. Where is integral time scale of the Lagrangian correlation. The fluctuation velocity $v_{f}^{\prime}$ and the eddy viscosity $\varepsilon_{t} / v$ of turbulent fluid are evaluated by the following empirical expressions, as given by Kallio and Reeks [11] and Davies [6], respectively,

$$
\sqrt{\overline{v_{f}^{\prime 2}}}=\sqrt{\overline{v_{f}^{\prime 2}} / u_{*}^{2}}=\frac{0.005 y^{+2}}{1+0.002923 y^{+2.128}} \text { for } 0<y^{+}<200 \text {, }
$$

$$
\begin{gathered}
\frac{\varepsilon_{t}}{v}=y^{+\left(4-y^{+0.08}\right)}\left(\frac{2.5 \times 10^{7}}{\operatorname{Re}}\right)^{-\frac{y^{+}}{400+y^{+}}} \times 10^{-3} \text { for all } \\
y^{+}=y u_{*} / v,
\end{gathered}
$$

where the Reynolds number $\operatorname{Re}=u_{\infty} d / v$ is based on the pipe diameter $d$ and the bulk fluid velocity $u_{\infty}$ in the central part of the pipe. As a result, the magnitude of $\bar{V}_{p c}^{+}$at the location of about one particle radius from the wall can thus be determined by substituting $y^{+}=r_{p}^{+}$into equations (30) and (31), and then by solving equation (29) for $\overline{v_{p}^{\prime 2}}$ and equation (26) for $\bar{V}_{p c}^{+}$.

The parameters of $Z_{c}=\exp \left(-y / \bar{H}_{c}\right)$ and $\bar{\psi}_{c}=\bar{C}-c_{\infty}$ are used in the development of an analytical solution for modelling parameter $\bar{C}$. With the aid of the additional parameters $\xi=2 \bar{H}_{c} Z_{c} / \sqrt{D_{p} \bar{\tau}}$, equations (23) and (24) take the forms

$$
\frac{d^{2} \bar{\psi}_{c}}{d \xi^{2}}+\frac{1+\frac{2 \bar{H}_{c} \bar{v}_{r}}{D_{p}}}{\xi} \frac{d \bar{\psi}_{c}}{d \xi}+\left(1-\frac{\frac{4 \bar{H}_{c}^{2}}{D_{p} \bar{\tau}}}{\xi^{2}}\right) \bar{\psi}_{c}=0
$$

Equation (32) are recognized as the Bessel differential equation of order $\sqrt{\left(2 \bar{H}_{c} / \sqrt{D_{p} \bar{\tau}}\right)^{2}+\left(\bar{H}_{c} \bar{v}_{r} / D_{p}\right)^{2}}$. Hence, an analytical solution that satisfies the corresponding boundary conditions can be obtained and expressed as

$$
\bar{\psi}_{c}=\left(c_{w}-c_{\infty}\right)\left[\frac{\xi}{2 \beta \exp \left(-r_{p} / 2 \bar{H}_{c}\right)}\right]^{-\bar{H}_{C} \bar{v}_{r} / D_{p}} \frac{J_{n}[\xi]}{J_{n}\left[2 \beta \exp \left(-r_{p} / 2 \bar{H}_{c}\right)\right]},
$$

where $\beta=\bar{H}_{c} / \sqrt{D_{p} \bar{\tau}}$ and $n=\beta \sqrt{\left(4 D_{p}+\bar{\tau} \bar{v}_{r}^{2}\right) / D_{p}}$. Reverting back into $y$ domain, equation (34) becomes 


$$
\frac{\bar{C}-c_{\infty}}{c_{w}-c_{\infty}}=\exp \left[\bar{v}_{r}\left(y-r_{p}\right) / 2 D_{p}\right] \frac{J_{n}\left[2 \beta \exp \left(-y / 2 \bar{H}_{c}\right)\right]}{J_{n}\left[2 \beta \exp \left(-r_{p} / 2 \bar{H}_{c}\right)\right]}
$$

Judging from a fact that the fluctuating properties of carrier fluid seen by suspended particles should increase with increasing the particle diameter, the wall boundary condition is actually applied at a distance of one particle radius from the wall, where the turbulence intensity is not zero. Hence, the particle deposition velocity of average rejuvenation period is defined as $\bar{v}_{d}=\bar{N} /\left.\left(c_{w}-c_{\infty}\right)\right|_{y=r_{p}}$ and can be approximately predicted by

$$
\bar{v}_{d}^{+}=\frac{\exp \left(-\frac{r_{p}^{+}}{2 \bar{H}_{c}^{+}} \bar{\tau}^{+}\right)}{\bar{\tau}^{+}} \sqrt{\frac{D_{p}}{v}}\left(\frac{J_{n-1}\left[\frac{2 \bar{H}_{c}^{+}}{\bar{\tau}^{+}} \sqrt{\frac{v}{D_{p}}} \exp \left(-\frac{r_{p}^{+}}{2 \bar{H}_{c}^{+}}\right)\right]}{J_{n}\left[\frac{2 \bar{H}_{c}^{+}}{\bar{\tau}^{+}} \sqrt{\frac{v}{D_{p}}} \exp \left(-\frac{r_{p}^{+}}{2 \bar{H}_{c}^{+}}\right)\right]}\right)-\frac{n}{2 \bar{H}_{c}^{+}} \frac{D_{p}}{v}+\frac{\bar{v}_{r}^{+}}{2}
$$

where $\quad \bar{H}_{c}^{+}=\bar{H}_{c} u_{*} / v \quad, \quad D_{p} / v=D_{B} / v+\varepsilon_{p} / v \quad$ and $\bar{v}_{r}^{+}=\bar{V}_{t h}^{+}+\bar{V}_{p c}^{+}+\bar{V}_{e}^{+}$. Herein, the algorithm of Bessel functions $J_{n}$ and $J_{n-1}$ is based on a code due to Sookne [22] that uses backward recursion with strict error control, and the Bessel function orders $n$, and $n-1$ have to be a positive integer.

\subsection{Physical Description of Charging Mechanisms}

The transport and deposition of aerosol particles under the influence of electrostatic force have become an important issue in many natural phenomena as well as in many aspects of aerosol technology and research. This rests on two basic facts that all natural or artificial aerosols are electrically charged, and that the force exerted on a moderately charged particle in an electric field is many times as compared with other forces that can be exerted on the aerosol particles. The high electric mobility of charged aerosols has been used in the development of a variety of electrostatic cleaners for commercial and residential applications, including electrically augmented filtration of contaminated aerosol particles and controlled placement of small particles from one surface to another. The amount of electric charges carried by airborne particles may significantly affect the adhesion of fine particles to specific sites, which may minimize the loss during manufacturing processes of the semiconductor industry or maximize deposition efficiency in the electro-photographic marking and the pharmaceutical industry. Generally, the amount of electric charges carried by the particle depends on the charging method. Bipolar ions bombard the particles, which will tend to attain an equilibrium charge state called the Boltzmann equilibrium. He and Ahmadi [23] denoted that Unipolar ions charge the particles through diffusion and field charging mechanisms, and that the aerosol particles reaching the maximum charge number at combined diffusion and field charging are said to be at saturation charge. As well as Turner and Fissan [24] and Hinds [9], they demonstrated that the number of charges carried by the airborne particles at the Boltzmann charge equilibrium is relatively low as compared with other charging mechanisms. Also, it has been shown that that, when the Boltzmann equilibrium and the uniform charge distribution are involved in theoretically analyzing the transport and deposition of particles in turbulent flows, the predicted values of deposition velocity for aerosol particles with saturation charge distribution are much larger than those of aerosol particles with Boltzmann equilibrium charge distribution in the presence of an external applied electric field [25-28].

When unipolar ions of a carrier fluid are formed, probably because of the ionizing radiation, the high voltage discharge or the high temperature, the ions with great diffusion tend to collide with any aerosol particles present quite rapidly, and will consequently become attached to the particles as a result of the electrostatic forces. The transport and deposition of particles which have been mixed with bipolar ions can be affected in various ways. The forces $F_{e}$ acting on a charged particle suspended in a gas near the conducting surface can be expressed as the form $[29,30]$

$$
\begin{aligned}
F_{e}= & q E(\text { Coulombic force })-\frac{q^{2}}{16 \pi \varepsilon_{o} y^{2}}(\text { Image force })+\frac{q E d_{p}^{3}}{16 y^{2}}(\text { Dielectrophoretic force }) \\
& -\frac{3 \pi \varepsilon_{o} d_{p}^{6} E^{2}}{128 y^{4}}(\text { Dipole }- \text { dipole force }),
\end{aligned}
$$

where $\varepsilon_{o}=8.859 \times 10^{-12} A s V^{-1} m^{-1}$ is the permittivity, $E$ is the electric field strength, and $y$ is the distance of the particle from the wall. The dielectrophoretic and the dipole-dipole forces on the induced dipole are caused by the gradient in image charge field and the interaction between the induced dipole and its image, respectively. These forces have been found to be negligible small compared to the Coulombic and the image forces, and the image force $F_{i}=q^{2} / 16 \pi \varepsilon_{o} y^{2}$ arises due to the induced charges on the wall and may become important under some specific conditions [31, 32]. Because 
the image force affects only a short range very near the surface, its effect is usually insignificant on particle deposition processes. Li and Ahmadi [27], therefore, pointed out that, when a charged particle is placed in an imposed electric field, the Coulombic force is the dominant force and can be determined by Coulombic law.

In this study, an applied electrical field is concerned with dilute particle concentration in which the space charge effect is ignored and, during the average rejuvenation period, the Coulombic force is considered as a dominated mechanism that causes the charged particle to move through the viscous wall region. Generally, the electrical characteristics of Coulombic force for an aerosol particle are specified by two properties in terms of the particle size and number of unit charge. For an aerosol particle carrying $n_{p}$ elementary units of electrical charge in an applied electrical field of intensity $E$, the total electrical charge by a particle is $q=n_{p} e$, then the Coulombic force due to the imposed field is $F_{e}=q E=n_{p} e E$. Hence, when the applied electrical force acting on the particle becomes equal to the viscous drag force, the average migration velocity attained by the particle can be obtained from Stokes law of drag force $F_{e}=3 \pi \mu d_{p} \bar{V}_{e} / C_{c}$ for spherical particles

$$
\bar{V}_{e}=\frac{n_{p} e E C_{c}}{3 \pi \mu d_{p}}
$$

where $e=1.602 \times 10^{-19} C$ is the electronic unit charge and $d_{p}$ is the particle diameter.

When aerosol particles are exposed to unipolar ions, most aerosol particles carry some electric charges and the charge levels depend on the charging mechanisms. The effects of the Coulombic force on the transport and deposition of particles are examined by two frequently encountered charging mechanisms in terms of the diffusion and the field charging mechanism. In the absence of an applied electric field, the aerosol particles become charged due to collision of the particles and the ions. This collision is primarily caused by the random thermal motion of the ions. Hence, it has been considered as a diffusion process that the gaseous ions diffuse toward the particle surface and impart their electric charges to the particle. According to Hinds [9], an approximate diffusion charging expression for the number of charges $n_{p}$ acquired by an initially neutral aerosol, which has been exposed to unipolar ions of an average concentration $N_{i}\left(\right.$ ions $\left./ \mathrm{m}^{3}\right)$ for a time duration $t$, is written as

$$
n_{p}=\frac{d_{p} K_{b} T}{2 K_{e} e^{2}} \ln \left(1+\frac{K_{e} d_{p} \bar{c} \pi e^{2} N_{i} t}{2 K_{b} T}\right),
$$

where $d_{p}$ is the diameter of aerosol particles and $K_{e}=9.0 \times 10^{9} \mathrm{~N} \cdot \mathrm{m}^{2} / \mathrm{C}^{2}$ is a constant of proportionality. Because aerosol particles are exchanging energy with the surrounding gas molecules, both the particles and the gas molecules have the same average kinetic energy, so that the mean thermal speed of the gaseous ions is calculated by $\bar{c}=\sqrt{48 K_{b} T / \pi^{2} \rho_{p} d_{p}^{3}}$. With an imposed electric field of intensity $E$, the ordered motion of the ions in the direction of the applied electric field increases the frequency of collision. The cause of this motion has been considered as the predominant charging mechanism that rapidly drives the unipolar ions onto the particle. Under these conditions, the random thermal motion of the ions may be ignored. If the ordered motion of the ions can be assumed to be confined along the electric line of force, the field charging equation for the maximum saturation number of charges $n_{p}$ acquired by a particle is given by

$$
n_{p}=\left(\frac{3 e_{p}}{e_{p}+2}\right) \frac{E d_{p}^{2}}{4 K_{e} e},
$$

where the relative permittivity or dielectric constant $e_{p}$ reflects the strength of electrostatic field produced in different materials by a fixed potential relative to that produced in a vacuum under the same conditions. It has been indicated that the dielectric constant $e_{p}$ is 1.00059 for air, 4.3 for quartz, 80.0 for pure water, and is infinite for conducting particles.

Actually, equations (39) and (40) are obtained by modifying the earliest expressions presented in Whitby and Liu [33]. Their calculated results demonstrated that the diffusion charging was a predominant mechanism for charging particles less than $0.2 \mu \mathrm{m}$ in diameter, even in the presence of an electrostatic field. As can also be seen from Hinds [9], for $N_{i} t>10^{12}$ ions $\cdot s / \mathrm{m}^{3}$ and $N_{i} t>10^{13}$ ions $\cdot s / \mathrm{m}^{3}$, equation (39) is shown to be accurate to within a factor of two for particles from 0.07 to $1.5 \mu \mathrm{m}$ and from 0.05 to $40 \mu \mathrm{m}$, respectively. However, the emphasis is given here on the physical understanding of the interaction between the dominant mechanisms of particle transport. For simplification, when the particles are mixed with unipolar ions in the presence of an external imposed electric field, they are assumed to be olive oil with a dielectric constant of $e_{p}=4.0$, and a value of $N_{i} t \cong 10^{12} \mathrm{~s} / \mathrm{m}^{3}$ is assumed. The effects of Coulombic force on the migration of such particles are treated as another spatially independent mechanism like the thermophoretic and turbophoretic mechanisms.

\section{Results and Discussions}

Adopting the Blasius resistance formula of the mean wall shear stress, $\bar{\sigma}_{w}=\rho u_{*}^{2}=\left(\mu+\rho \varepsilon_{t}\right) \partial \bar{U}_{f} /\left.\partial y\right|_{y=0}$, the proposed relationship for the mean residence time $\bar{\tau}$ between two successive eddies can be obtained on the basis of equation (8) and expressed as following form to brings out the dependence of $\bar{\tau}^{+}$on fluid velocity, 


$$
\bar{\tau}^{+}=u_{\infty}^{+} \sqrt{1+\varepsilon_{t} / v}\left(\frac{J_{2 \omega-1}[2 \omega]-J_{2 \omega}[2 \omega]}{J_{2 \omega}[2 \omega]}\right)
$$

where undetermined parameter $\omega=\bar{H} / \sqrt{\left(v+\varepsilon_{t}\right) \bar{\tau}}$ acts as a dominating parameter for characterizing the wall-normal inertia of turbulent fluctuation fractions intermittently moving from the turbulent core to near the wall. A comparison of $\bar{\tau}^{+}$ with experimental data $[13,17]$ is reestablished with an apparently more plausible assumption that the bulk mean fluid velocity $u_{\infty}^{+}=u_{\infty} / u_{*}$ is determined by coupling the average velocity profile with the familiar logarithmic low $\bar{U}_{f}^{+}=2.5 \ln \left(y^{+}\right)+5.5$ of the intermediate zone. Based on the Prandtl formula for the friction factor $f=2\left(u_{*} / \bar{U}_{f}\right)^{2}$, the logarithmic velocity profile can be written as $u_{\infty}^{+}=4 \sqrt{2} \log \left(2 \sqrt{2} r^{+}\right)-0.4 \sqrt{21}$, where the tube radius $r^{+}=\operatorname{Re} / 2 \sqrt{f / 2}$ and the friction factor can accurately be estimated by $f=0.07725(\log \mathrm{Re} / 7)^{-2}$ [34]. Figure 1 shows that the calculations obtained on the basis of this analysis for $\bar{\tau}^{+}$are dramatically changed with $\omega$ and trend to near a constant at high $\mathrm{Re}$. The value of $\omega=4.5$ seems to be a correct value because $\bar{\tau}^{+}$ranged $14.779 \leq \bar{\tau}^{+} \leq 16.897$ is quite satisfactory with the visual observation [12, 13], $14.0 \leq \bar{\tau}^{+} \leq 17.0$ for $2.0 \times 10^{4}<\operatorname{Re}<5.5 \times 10^{4}$. Also, $\bar{\tau}^{+} \approx 18.0$ at high $\mathrm{Re}$ is in good agreement with the experimental data [17-19].

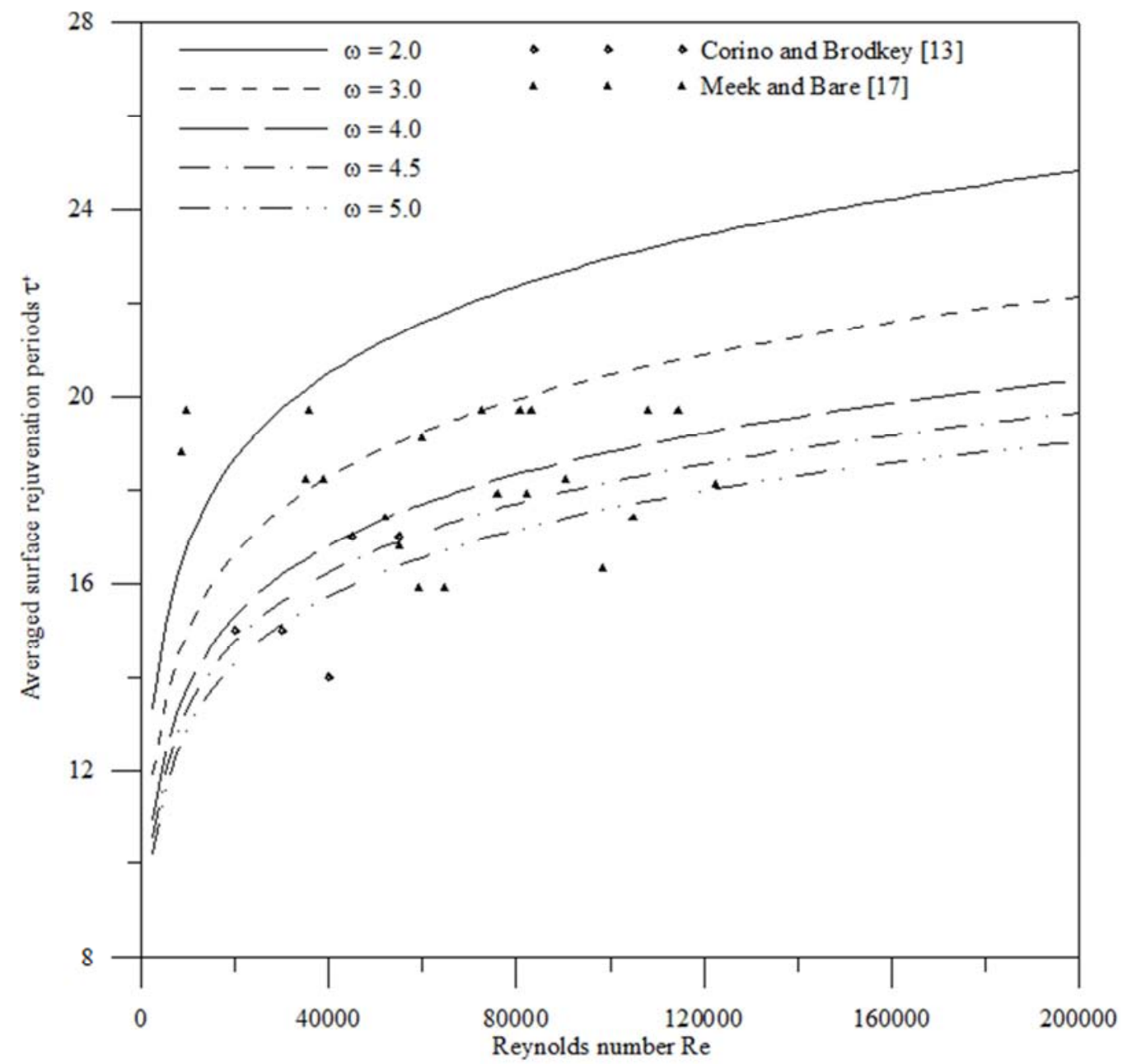

Figure 1. Averaged surface rejuvenation period as a function of Reynolds number for specified transfer parameter $\omega\left(=\bar{H} / \sqrt{\left(v+\varepsilon_{t}\right) \bar{\tau}}\right)$.

Equation (36) has been written in a form to bring out the dependence of $\bar{v}_{d}^{+}$on $\bar{H}_{c}^{+}$. Hence, an accurate prediction of $\bar{v}_{d}^{+}$would be directly related to an accurate prediction of influences on $\bar{H}_{c}^{+}$by the dominant deposition mechanisms.
For the given order $n \quad\left(=\bar{H}_{c} / \sqrt{D_{p} \bar{\tau}} \sqrt{\left.\left(4 D_{p}+\bar{\tau} \bar{v}_{r}^{2}\right) / D_{p}\right)}\right.$, which can be treated as a transport parameter to link the transport and deposition of immersed particles with the contributions of dominant deposition mechanisms, the relative distance $\bar{H}_{c}^{+}$of particle concentration boundary 
layer at the interface can be approximately estimated by

$$
\bar{H}_{c}^{+}=\frac{n \bar{\tau}^{+} D_{p} / v}{\sqrt{4 D_{p} / v+\left(\bar{\tau}^{+} \bar{v}_{r}^{+}\right)^{2}}} .
$$

In the absence of thermophoretic and electric effects, only turbulent diffusivity $\varepsilon_{p} / v$ of particles is left unspecified, and the drift velocity $\bar{v}_{r}^{+}$becomes dominated by the convective velocity $\bar{V}_{p c}^{+}$which depends solely upon the ability that the particles respond to the near-wall velocity fluctuations. Hence, the calculations for $\bar{v}_{d}^{+}$with the aid of following equations (43) and (44) is completed by providing a prescription for $\varepsilon_{p} / v$. The variation of $\bar{v}_{d}^{+}$as a function of $\tau_{p}^{+}$is graphically represented in figure 2 together with the predictions obtained by free-flight theories [5-7]. The measured deposition velocities [10] and the numerical experiment results [11] are also plotted in this figure. This comparison with the available experimental data and theoretical predictions will permit a determination of empirical correlations for $\varepsilon_{p} / v$.

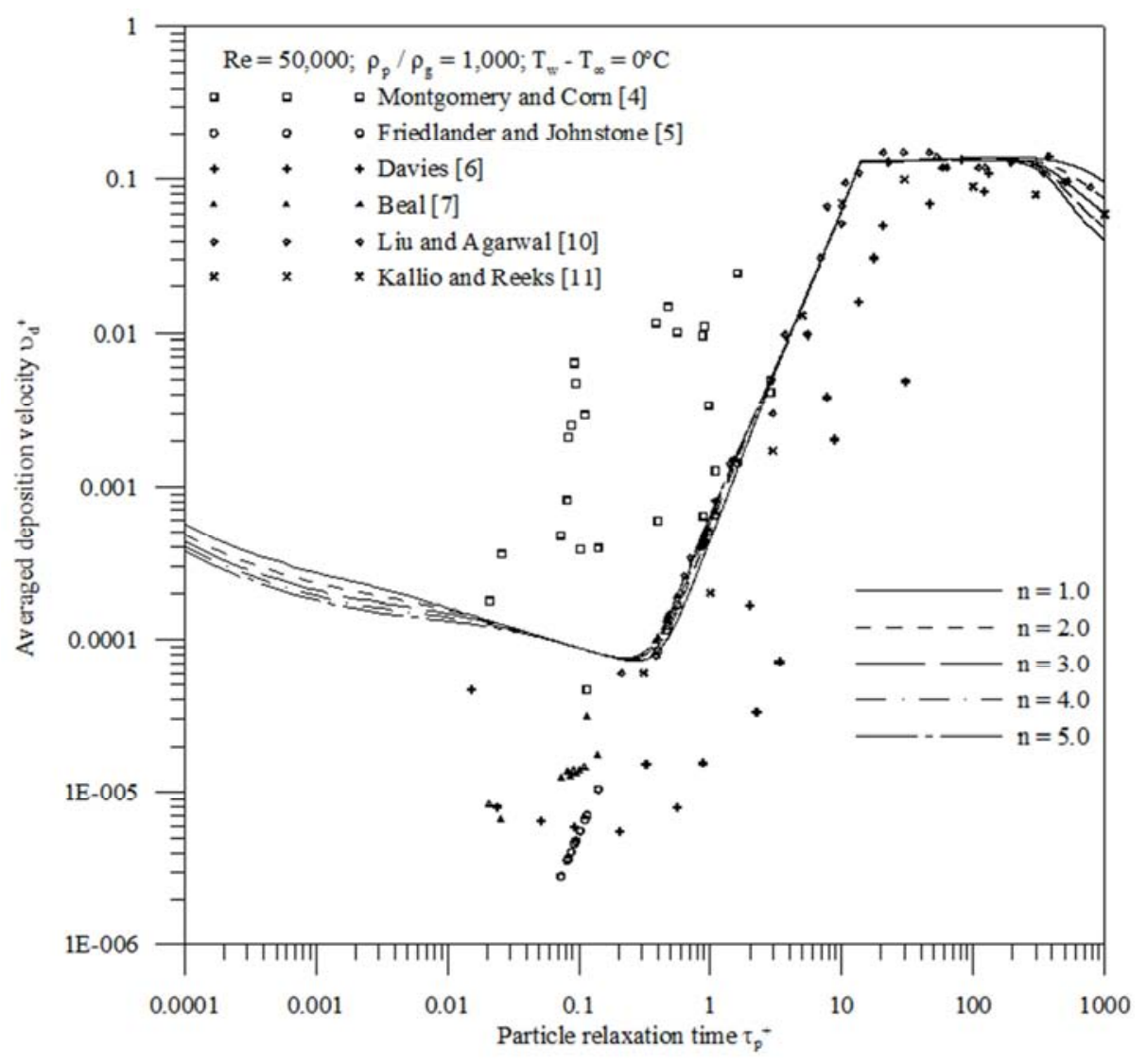

Figure 2. Comparison of $\bar{v}_{d}^{+}$vs. $\tau_{p}^{+}$dependence with available experimental data and theoretical predictions under various values of transferred parameter $n\left(=\beta \sqrt{\left.\left(4 D_{p}+\bar{\tau} \bar{v}_{r}^{2}\right) / D_{p}\right)}\right.$.

The empirical curve-fit used in free-flight theories for the variation of $\varepsilon_{p} / v$ as a function of the wall-coordinate $y^{+}$ is modified by changing the adjustable constants, which are tested up to a Bessel function order $n=11$. It has been found that the best fit can be attained for Bessel function orders up to $n=11$, when the expressions of $\varepsilon_{p} / v$ in the viscous wall region are prescribed as the form

$$
\begin{gathered}
\frac{\varepsilon_{p}}{v}=\left(0.0003 n^{2}+0.0057 n+0.0066\right) \tau_{p}^{+3} y^{+3} \text { for } y^{+}<0.25, \\
\frac{\varepsilon_{p}}{v}=0.0777 n^{-0.728} \bar{H}_{c}^{+} \text {for } y^{+}>0.25
\end{gathered}
$$

The lower boundary of $\varepsilon_{p} / v$ in the neighborhood of the wall is taken into account for a limit that the immersed particles could be identified as carrier fluid, and the inclusion of $\tau_{p}^{+3}$ in the proportional coefficient is associated with the influence of particle inertia on the dissipation of particles by fluid turbulence. However, for particles bigger than a critical size, the inertial influence will become such that the fluid velocity fluctuations given to them by carrier eddies are not dissipated rapidly in the near-wall region. A combination of both the particle inertia and the turbulent fluctuation intensity is then considered to be responsible for the particles to coast across the viscous sublayer, and the degree of inertial enhancement in particle deposition due to this combined effect 
is characterized by equation (44). For smaller particles, because of the decreased Brownian diffusion effect, $\bar{v}_{d}^{+}$ deceases monotonically with increased $\tau_{p}^{+}$. After passing through a minimum, $\bar{v}_{d}^{+}$rises dramatically by several orders of magnitude as $\tau_{p}^{+}$increases, at which the dependence of $\varepsilon_{p} / \nu$ on $\bar{H}_{c}^{+}$becomes more prominent in the presence of turbulent fluctuations near the wall. However, the subsequent increase in $\bar{v}_{d}^{+}$is not monotonic. Just like the maximum growth thickness for $\bar{H}_{c}^{+}$are restricted by the periodically developing boundary layer approximately around $y^{+} \approx 30$, there is an upper limit of $\bar{H}_{c}^{+}$for reflecting the effect of turbulent fluctuation intensity on $\varepsilon_{p} / v$. This seems to be applicable because the region of $y^{+} \geq 30$ is often assumed to be a uniformly mixed turbulence core, where the turbulent diffusivity of particles is generally small and negligible.

With the consideration of an additional turbophoresis generated by the fluctuating velocity gradient of particles, as illustrated by $\bar{v}_{d}^{+}$curves of figure 2 , the peak value of $\bar{v}_{d}^{+}$ remains nearly constant with increased $\tau_{p}^{+}$in the particle inertia-moderated regime and exhibits a tendency to be decreased as $\tau_{p}^{+}$becomes very large. This trend in $\bar{v}_{d}^{+}$can easily be conceivable from the reduced momentum equation (16) for the convective acceleration of particles, which arises primarily due to an imbalance in the viscous drag resistance and turbophoresis. As can be noted in figures 3 and 4, when the inertia of particles is sufficiently large, the fluctuating velocity gradient of immersed particles in the vicinity of the wall becomes larger than that of carrier fluid, and the turbophoresis stemmed from the gradient in particle fluctuation velocity grows and induces a convective velocity toward the wall, respectively. Especially, for particles in the particle inertia-moderated regime, the viscous dreg resistance loses importance, and the turbophoretic force in the vicinity of the wall not only enhances the convective flux through the increased convective velocity, but also simultaneously attenuates the diffusive flux through decreased turbulent diffusivity of particles. Because the enhancement in convective flux is larger than the reduction in diffusive flux, hence, the total deposition flux remains relatively insensitive to the changes of $\tau_{p}^{+}$in the particle inertia-moderated regime. Also, since the fluid velocity fluctuations seen by the inertia-dominated particles should increase with the increase of their inertia, the particle response to the wallward fluid turbulence would be completely attenuated as $\tau_{p}^{+}$is increased beyond a certain limit. This is one of major factors responsible for the eventual decrease in $\bar{v}_{d}^{+}$with increasing $\tau_{p}^{+}$when $\tau_{p}^{+}$is very large. It can be concluded from the above results that the particle inertia can not always manifest itself as an increased diffusivity in the wall-bounded shear layer, and that the inclusion of turbophoresis serves to enhance the relative motion of the immersed particles to the carrier fluid.

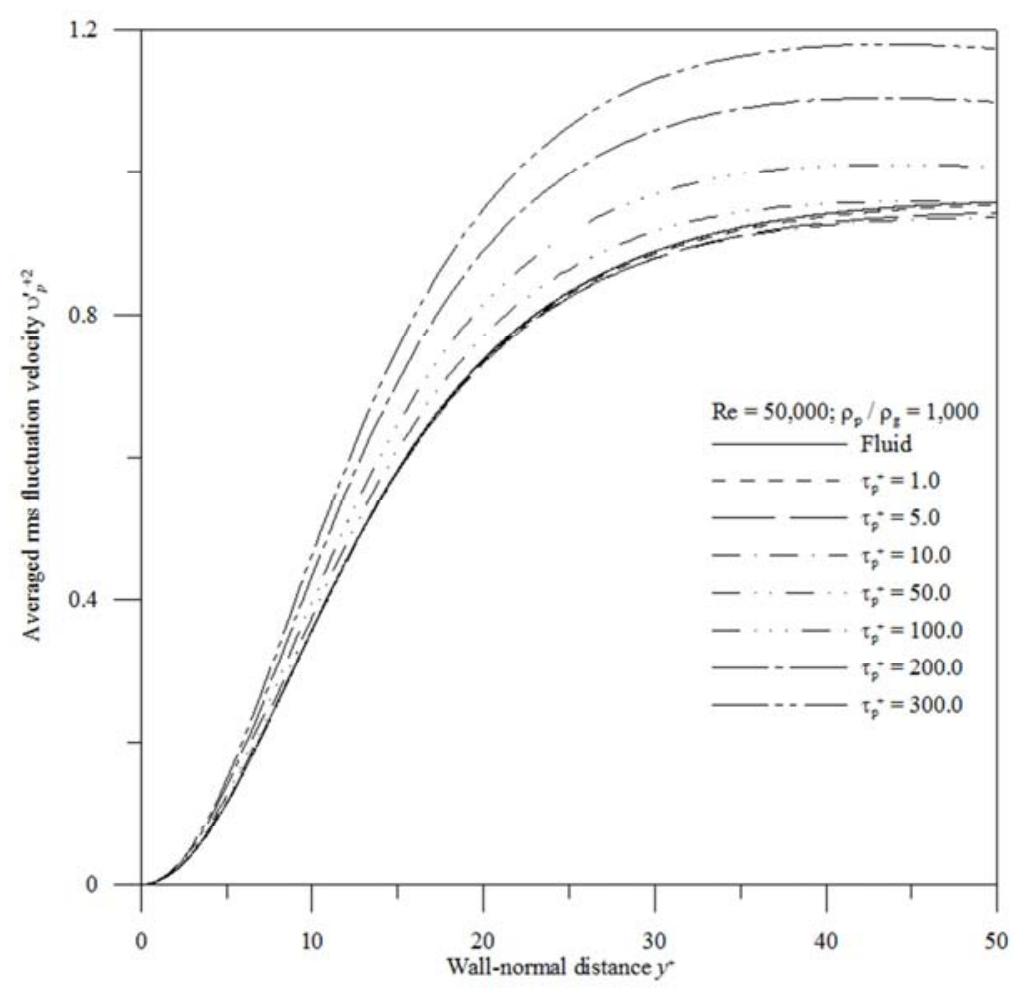

Figure 3. Distribution of averaged fluctuating velocity near the wall for various particle relaxation times (particle inertia). 


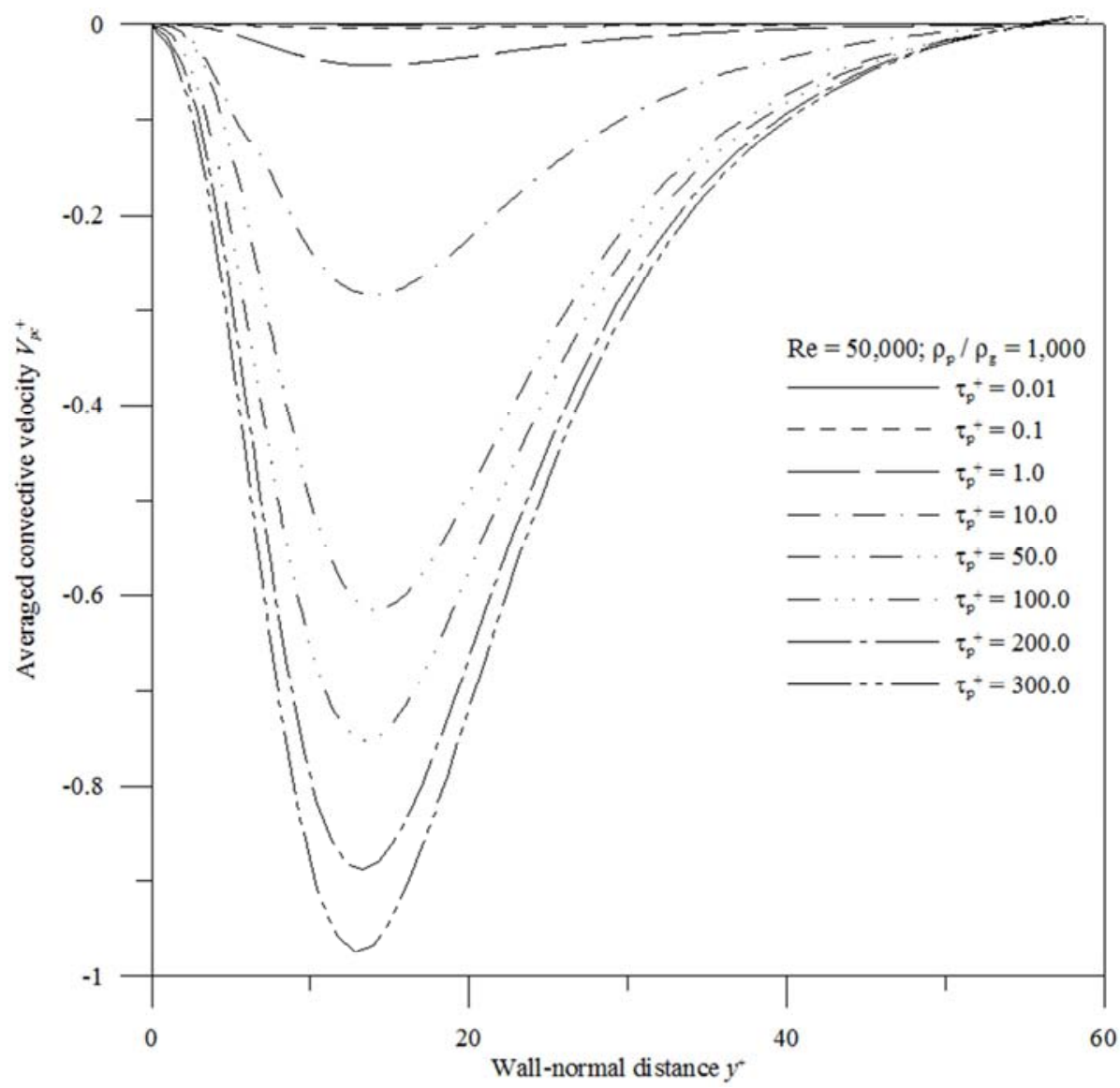

Figure 4. Distribution of averaged convective velocity near the wall for various particles relaxation times (particle inertia).

The predictions obtained by the present analysis for $\bar{v}_{d}^{+}$ show a good agreement with available experimental and numerical data. Also, the close agreement over a wide range of $\tau_{p}^{+}$may be regarded as supporting evidence for the adequacy of the present formulation. Although the difference between simulated $\bar{v}_{d}^{+}$curves is not large for each Bessel function order, the subsequent analysis will simply set $n=3$ for all particle sizes because, for particles with $\tau_{p}^{+}>100$, the predicted trend of $\bar{v}_{d}^{+}$accompanied by the maximum value of $\bar{H}_{c}^{+} \approx 25.515$ does better job of fitting the experimental data than others. In order to further verify the adequacy of equations (43) and (44), the values of $\bar{v}_{d}^{+}$calculated by taking the different correlations into account for $\varepsilon_{p} / v$ are depicted in figure 5 without the effects of thermophoresis and electric force. As was first mentioned in Friedlander and Johnstone [5], the basic framework of free-flight model is based on the assumption that the particle diffuse towards a stopping distance $S$ close to the wall, at which point the particles will effectively break away from the containing eddies and embark on a free-flight with a given initial velocity $v_{i}$ to coast across the viscous sublayer. In their calculations, the effect of Brownian diffusion is neglected, and the use of the fluid eddy viscosity $\varepsilon_{t} / v$ to couple with the free-flight velocity $v_{i}=0.9 u_{*}$ at stopping distance $S=v_{i} \tau_{p}$ is directly related to the large increase of $\bar{v}_{d}^{+}$in the turbulent diffusion-eddy impaction regime. As can be noted, this model yielded a reasonable agreement with experimental data in an intermediate range of particle relaxation time, but not for particles of high inertia. The same basic framework of free-flight model was used in Davies [6] and Beal [7] with different assumptions for the particle velocity at the start of free-flight. Beal [7] proffered that the axial fluid velocity at a particle radius from the wall can be taken into account for the free-flight velocity, while Davies [6] made an apparently more plausible assumption of $v_{i}=\sqrt{\overline{v_{f}^{\prime 2}}}$ for particle migration across the relatively stagnant fluid next to the wall. It is interesting to note that the predictions for $\bar{v}_{d}^{+}$ obtained on the basis of Davies's model appear to be lower than the experimental measurements, but those obtained by Beal [7] are similar to the original work of Friedlander and Johnstone [5]. Furthermore, the calculations for $\bar{v}_{d}^{+}$are modified by introducing the correlations of $\varepsilon_{p} / v$ proposed by Sehmel [35] into the analysis instead of equations (43) and (44). It is found that, because the increase in $\varepsilon_{p} / v$ with the wall-normal distance $y^{+}$leads to a steep rise in $\bar{v}_{d}^{+}$curve, the values of $\bar{v}_{d}^{+}$for particles in the particle 
inertia-moderated regime become much higher than the measured deposition velocities. In order to improve the predictions of Davies's model, Liu and Ilori [8] has proposed a constitutive relation for particle eddy diffusivity $\varepsilon_{p} / v$, by adding an additional factor $\overline{v_{f}^{+2}} \tau_{p}^{+}$to account for the enhanced deposition by inertia. The calculations obtained on the basis of equation (1) are also plotted in this figure for comparison. Apparently, the additional factor $\overline{v_{f}^{\prime 2}} \tau_{p}^{+}$does not reflect the difference between the particle and fluid fluctuation velocity, and consequently results in a monotonic increase in $\bar{v}_{d}^{+}$with increasing $\tau_{p}^{+}$due to an indefinite increase in $\varepsilon_{p} / v$ with $\tau_{p}^{+}$.

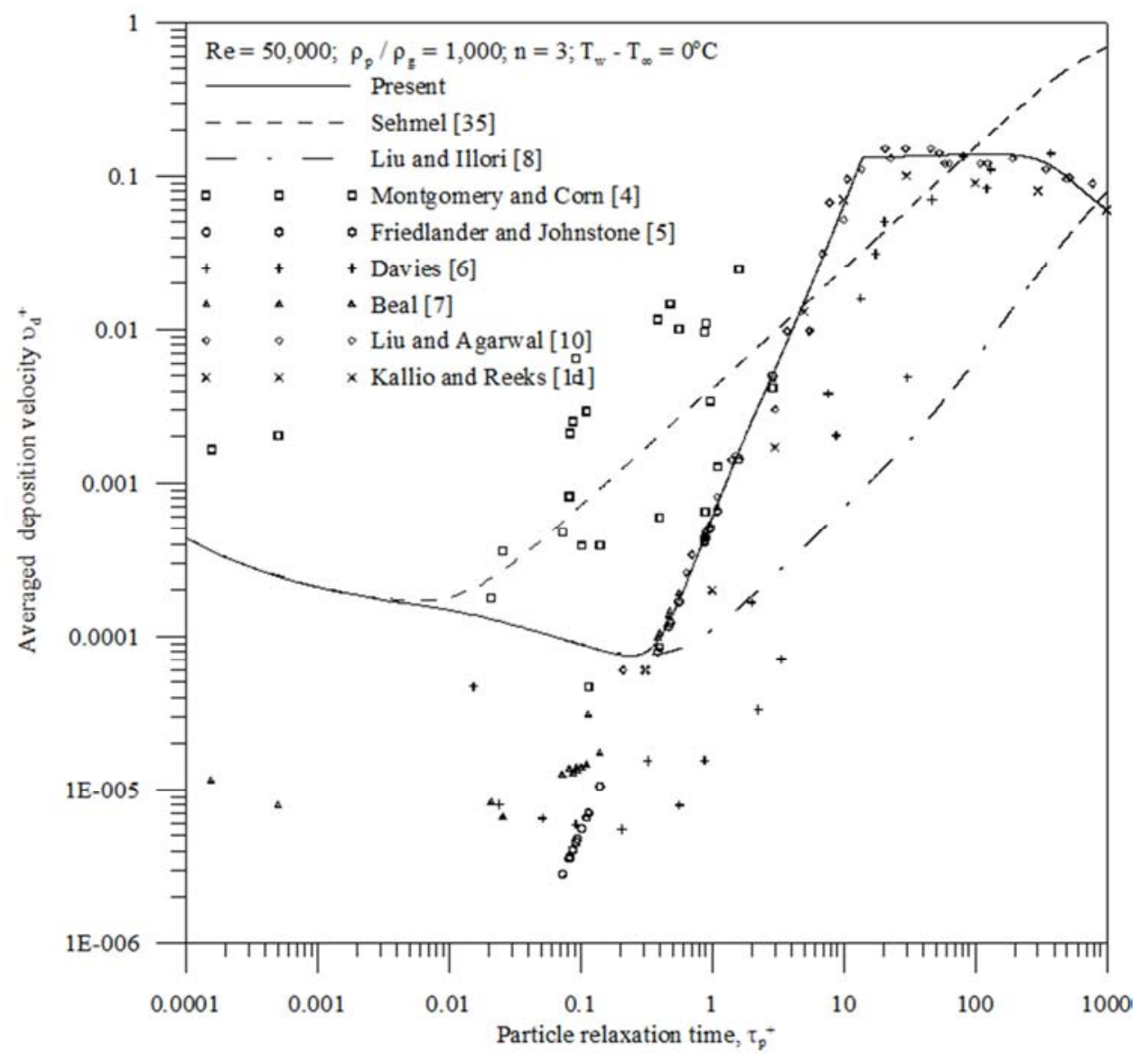

Figure 5. Calculations for averaged particle deposition velocity under different correlations of turbulent diffusivity of particles.

In the absence of external imposed electric field, the proposed relationship for $\bar{v}_{d}^{+}$obtained on the basis of the surface rejuvenation model has been testified to be valid for Brownian diffusion becoming appreciable, and for the particles having considerable inertia that they fail to respond to the velocity fluctuations of carrier fluid. In comparison with the experimental data [10] and numerical predictions [11], the close agreement in the trend of $\bar{v}_{d}^{+}$obtained by the proposed method gives a strong support to the proposed approach for particle deposition problem. Therefore, when the external electric filed is merged into the turbulent flow, this characteristic dependence of $\bar{v}_{d}^{+}$on $\tau_{p}^{+}$can reasonably be used for clarifying the deposition behaviour of charged particles. In the following analysis, the aerosol particles are assumed to be produced by olive oil with a dielectric constant of $e_{p}=4.0$, and charged by unipolar ions through different mechanisms, the diffusion charging (DC), the field charging
(FC), and the saturation charge distribution as well as the combined diffusion and field charging (DC+FC). The Coulombic force due to the imposed electric filed is considered to be directed towards the wall, and once the particles reach the wall surface they will stick to it. For a fixed electric field intensity of $E=20 \mathrm{kv} / \mathrm{m}$, the variations of $\bar{v}_{d}^{+}$with $\tau_{p}^{+}$for particles that carry charges under the action of different charging mechanisms are represented in figure 6 , where the influences of Coulombic force are examined based on there different charging mechanisms. The resulting deposition velocity of particles in the absence of electric effects is also shown in this figure for comparison. When the diffusion charging (DC) is considered as the only charging mechanism, the deposition velocity of submicrometer-sized particles increases rapidly in comparison to that of neutral particles, while for particles with $\tau_{p}^{+}>0.003$, the amount of increase is small and negligible. This trend in $\bar{v}_{d}^{+}$ 
emphasizes the fact that the diffusion charging is caused by the random collisions between the particles and ions due to the Brownian motion process, and therefore the enhancement in the unassisted deposition velocity occurs due to the Coulombic force effect only when the deposition flux towards the wall is entirely predominated by the Brownian diffusion. However, there certainly exists a significant quantitative difference between the deposition curves if one includes the effect of filed charging (FC). As given in equation (29), the number of charges carried by a particle increases in directly proportion to the square of particle diameter. Accordingly, the Coulombic force increases significantly as particle diameter increases. It can be observed that, when the field charging is the only concern, the predictions for $\bar{v}_{d}^{+}$always remain much higher than the values of neutral particles, and this discrepancy is considerably more pronounced as compared to that for particles with the diffusion charge. This demonstrates that the Coulombic force can lead to a considerable reduction in the diffusional deposition mechanism of particles through the enhanced drift velocity in the near-wall region, and that the different between the amounts of charges carried by particles can be very significant in modifying the deposition velocities.

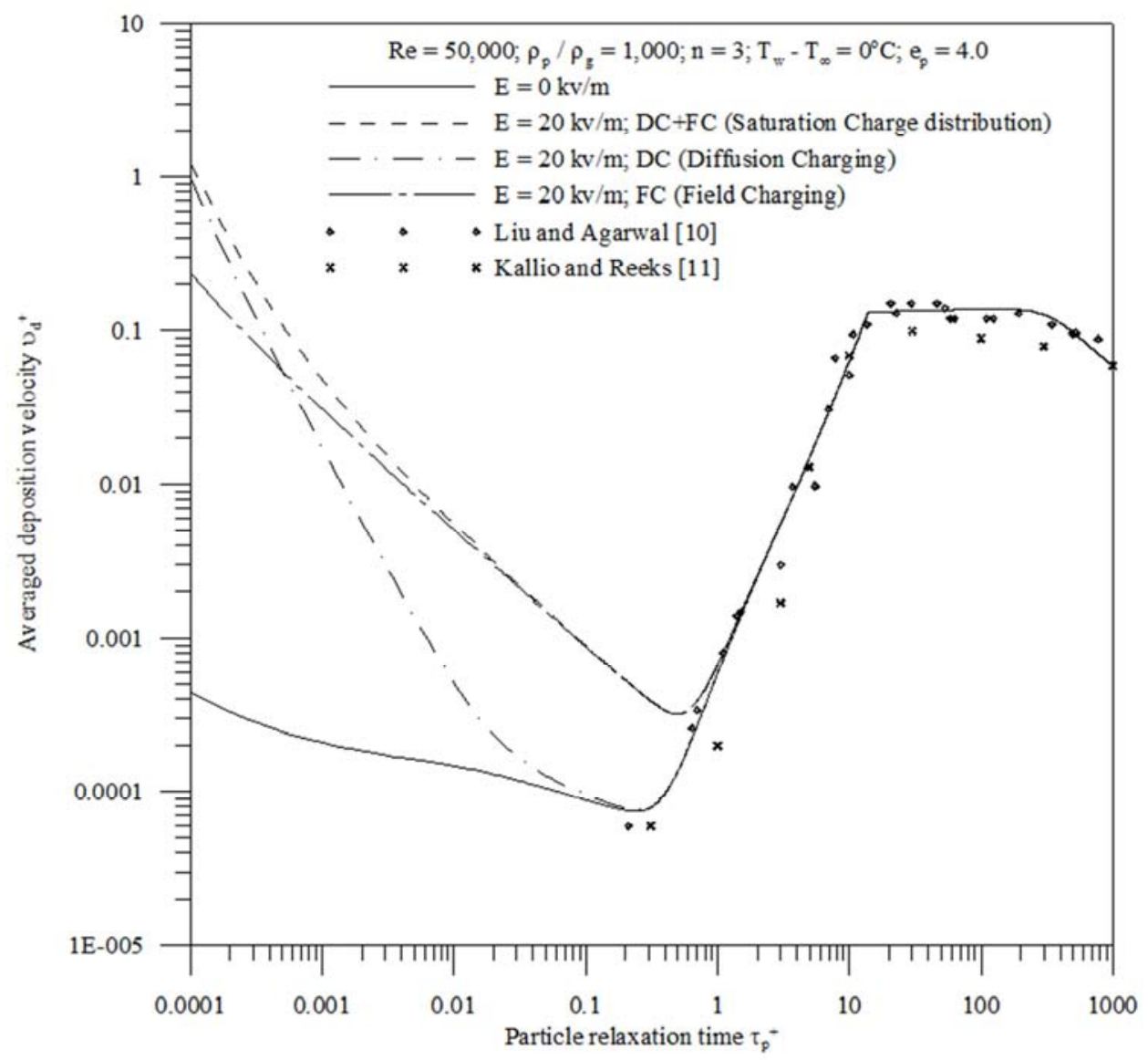

Figure 6. Dependence of Coulombic force on charging method in the presence of an external imposed electric filed.

The saturation charge distribution $(\mathrm{DC}+\mathrm{FC})$ may generally be the most representatively charging mechanism for particles reaching the maximum charge number at combined diffusion charging and filed charging. Analogously as the dependence of diffusion charging and field charging upon the particle inertia $\left(\tau_{p}^{+}\right)$, the relative importance of both charging mechanisms on $\bar{v}_{d}^{+}$can clearly be appreciated from the $\bar{v}_{d}^{+}$ curve simulated for particles at the saturation charge distribution. As can be observed, there is an overlap between two $\bar{v}_{d}^{+}$profiles, such as that the saturation charge overlaps the functions of the field charging already in existence. This provides an optimum range of $\tau_{p}^{+}$for reflecting the contribution of field charging to the saturation charge distribution. Because the deposition velocities in this $\tau_{p}^{+}$ range are shown to be much higher than the values predicted for particles with the diffusion charge, hence, it can be concluded that one of most striking effects of aerosol particle interaction with an imposed electric field is an existence of field charging region. However, there is a significant decrease in the effect of field charging with continued reduction in $\tau_{p}^{+}$, separating the $\bar{v}_{d}^{+}$curves with heightening $\bar{v}_{d}^{+}$for particles at the saturation charge distribution. The reason is that both diffusion and field charging mechanisms would play comparable roles within the field charging region and then be succeeded by the effect of diffusion charging. As the results indicate, the Coulombic force caused by diffusion 
charging mechanism increases with decreasing $\tau_{p}^{+}$, which subsequently makes $\bar{v}_{d}^{+}$increase in the submicrometer-size range.

Figure 7 displays the simulated $\bar{v}_{d}^{+}$curves for particles that carry the saturation charge under the different electric field strengths. A comparison with results of neutral particles, the effect of Coulombic forces is clearly seen as the particles continue to accelerate towards the wall despite the reduction of the diffusional deposition mechanism, and thus the increase in Coulombic force effect with increased electric field strength can lead to significant deviations from the neutral particle deposition curve. This is evident from a rather wide distribution of $\bar{v}_{d}^{+}$coupled with a wider range of $\tau_{p}^{+}$ being eliminated from the turbulent diffusion-eddy impaction regime in the presence of high electric field strength. It should be noted that the turbulent diffusivity of particles $\left(\varepsilon_{p} / v\right)$ increases with increasing $\tau_{p}^{+}$in the turbulent diffusion-eddy impaction regime and eventually dominates over the Coulombic force effect beyond a certain limit, according to the strength of imposed electric field. The particles with their high inertia then have sufficient wallward momentum to coast across the viscous sublayer and deposit on the wall regardless of the imposed electric field intensity. On the other hand, as $\tau_{p}^{+}$is decreased from this limit, an interesting observation is that $\bar{v}_{d}^{+}$initially decreases with decreased $\tau_{p}^{+}$, goes through a minimum and then increase rapidly with any further decrease in $\tau_{p}^{+}$. As was mentioned in Flagan and Seinfeld [36], this trend in $\bar{v}_{d}^{+}$is directly related to the significant modification of $\bar{v}_{d}^{+}$vs. $\tau_{p}^{+}$dependence, and can be attributed to the decreasing charge carried by particles of smaller size and the increase of mobility with decreasing size resulting in an offsetting of the reduced charge.

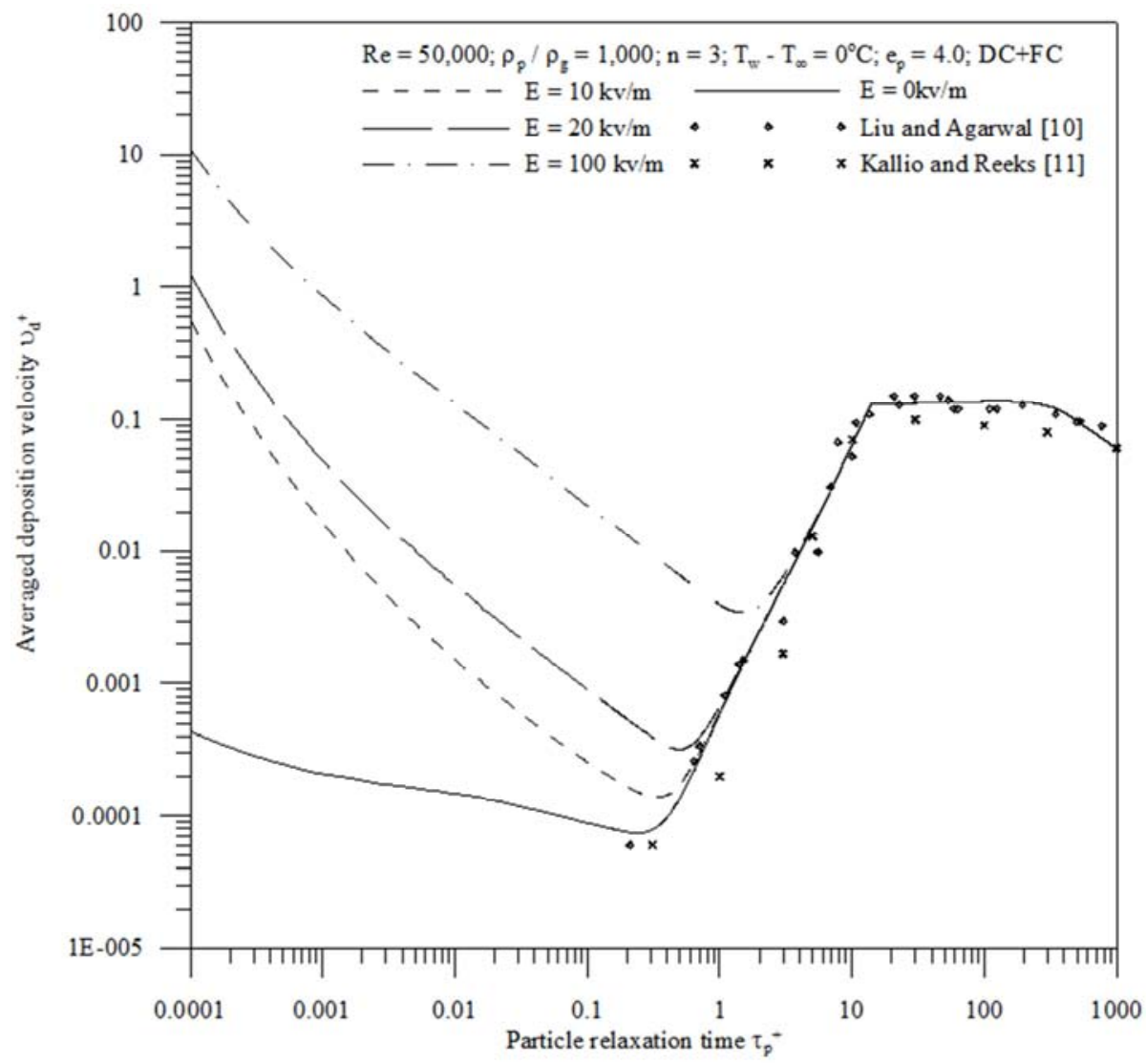

Figure 7. Effect of Coulombic forces on deposition velocities for particles at saturation charge distribution under various strengths of external imposed electric field.

Furthermore, for particles with saturation charge suspended in the various Reynolds number flows, the predicted trends of $\bar{v}_{d}^{+}$under a fixed electric field intensity of $E=20 \mathrm{kv} / \mathrm{m}$ are plotted in figure 8 as a function of $\tau_{p}^{+}$. The results for neutral particles are also shown in this figure for comparison. It should be reminded that, based on the surface rejuvenation concept, the velocity gradient of an incoming wallward eddy is gradually decelerated in a progressively developing viscous motion along the interface until a subsequent penetrating eddy, and the established turbulent boundary layer during the average rejuvenation period consists of a viscous sublayer between the uninfluenced thin wall-layer and the outer layer above viscous sublayer. As the fluid velocity increases, the momentum carried across the streamlines of the average flow by turbulence actually causes steeper velocity gradient with 
the wall in a very short distance. Hence, the particles moving against the deeper turbulence penetrations can reach much more quickly the region very near the wall, where the Brownian diffusion $\left(D_{B} / v\right)$ of neutral particles is relatively significant. As illustrated by $\bar{v}_{d}^{+}$curves for neutral particles, $\bar{v}_{d}^{+}$increase with the increase of Reynolds number, and the shift in a minimum of $\bar{v}_{d}^{+}$towards larger $\tau_{p}^{+}$occurs due primarily to $\varepsilon_{p} / \nu$ and $D_{B} / \nu$ being comparable. Although changes in $\bar{v}_{d}^{+}$curves with Reynolds number are similar to those observed of neutral particles, however, the values of $\bar{v}_{d}^{+}$at the same Reynolds number flow remain higher than those predicted in the absence of the Coulombic force effects. Also, since the increase of Reynolds number has a bigger influence on $\bar{v}_{d}^{+}$of small particles than that of large particles, the increment of this increase tendency is found to become more pronounced for the micrometer-sized and smaller particles. It is evident that, although the ascendant tendency observed for $\bar{v}_{d}^{+}$curves of particles at the saturation charge distribution is similar to that of neutral particles, however, the steep rise in $\bar{v}_{d}^{+}$curves is not caused by this but rather by the effects of Coulombic force. This can easily be conceivable from figure 7 . Because the effects of diffusional deposition mechanism have been found to become comparatively less significant with the increase in the electric field intensity, the effect of the near-wall turbulent flow is probably weakened by the presence of relatively high electric field strength, and in consequence the particles with saturation charge can be deposited on the wall readily regardless of the turbulence intensity.

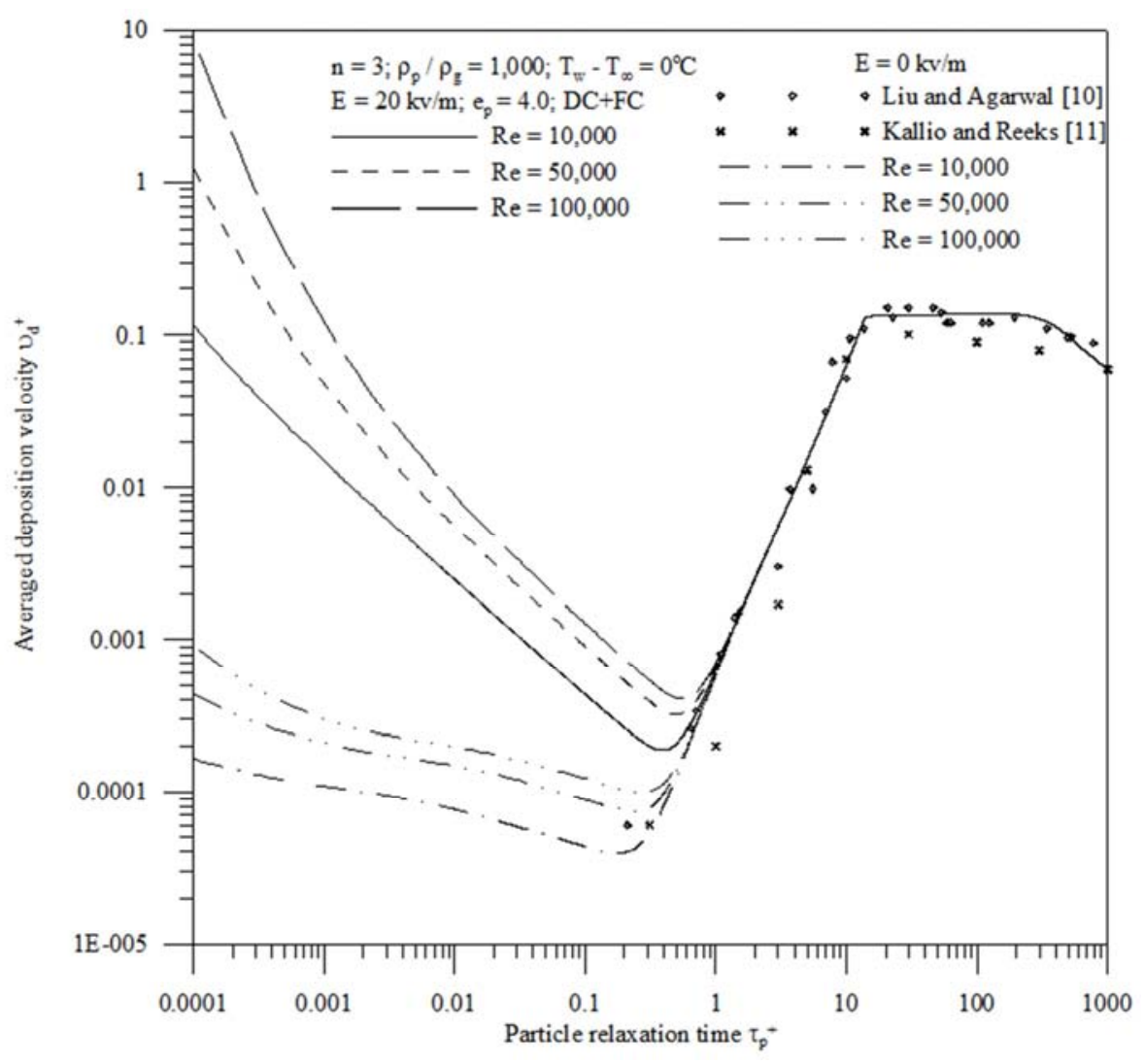

Figure 8. Variations of deposition velocity with Reynolds number for particles at saturation charge distribution under an external imposed electric field.

When the variations in thermal intensity between colder and hotter region of containing fluid are maintained, the thermophoresis arising from the gradient of this thermal intensity has been recognized to be very significant in modifying the deposition velocities. Wang et al. [37] has studied the thermophoretically driven migration of submicron particles when flowing around droplets under different conditions by direct numerical simulation. The thermophoretic deposition efficiency of single droplets has been found to be the basis for analyzing industrial spraying and wet deposition process of fine particles. Eslamian et al.
[38] demonstrated that using a nanofluid in bottom-heated laminar natural convection results in a considerable increase in heat transfer rate and thermophoresis force is a significant contributor to heat transfer augmentation, particularly for high Ra numbers. In experimental study of Zhou et al. [39], a microfluidic device was used to directly observe and quantitatively characterize the thermophoretic behaviors of charged hydrophobic polystyrene particles within a wide diameter range from $100 \mathrm{~nm}$ to $5 \mathrm{~lm}$ dispersed in deionized water. As a result of particle size effect on the thermophoresis of such particles, the increase in particle size was found to not 
only alter the direction of thermophoretic motion, but also linearly increase the thermophoretic velocity.

As given by Talbot et al. [40], the thermophoretic force per unit particle mass is usually transformed into the corresponding thermophoretic velocity $V_{t h}=K_{t h} v(\partial \ln T / \partial y)$, where the thermophoretic coefficient $K_{t h}$ is taken into account for the effect of the temperature gradient inside the particle. Also, based on a constitutive relation given by equation (9) for the fluid temperature distribution of average time domain, the thermophoretic velocity $\bar{V}_{t h}^{+}$at location of about one particle radius from the wall can be evaluated by

$$
\bar{V}_{t h}^{+}=\frac{K_{t h}}{\bar{\tau}^{+} \sqrt{\frac{\alpha}{v}+\frac{\varepsilon_{t}}{v}}}\left(\frac{T_{w}-T_{\infty}}{T_{\infty}}\right) \frac{\left(\exp \left(\frac{-y^{+}}{2 \bar{H}^{+}}\right) J_{2 \vartheta-1}\left[2 \vartheta \exp \left(\frac{-y^{+}}{2 \bar{H}^{+}}\right)\right]-J_{2 \vartheta}\left[2 \vartheta \exp \left(\frac{-y^{+}}{2 \bar{H}^{+}}\right)\right]\right)}{\left(J_{2 \vartheta}[2 \vartheta]+\left(\frac{T_{w}-T_{\infty}}{T_{\infty}}\right) J_{2 \vartheta}\left[2 \vartheta \exp \left(\frac{-y^{+}}{2 \bar{H}^{+}}\right)\right]\right)},
$$

where $\vartheta=\bar{H} / \sqrt{\left(\alpha+\varepsilon_{t}\right) \bar{\tau}}$ and $\alpha$ is thermal diffusivity of carrier fluid. With the Stokes-Cunningham slip correction factor $C_{f}=1+K_{n}\left[1.257+0.4 \exp \left(-1.1 / K_{n}\right)\right]$ given by Hinds [9], the thermophoretic coefficient $K_{t h}$ can be written as

$$
K_{t h}=\frac{2 C_{f} C_{s}\left(K_{g} / K_{p}+C_{t} K_{n}\right)}{\left(1+3 C_{m} K_{n}\right)\left(1+2 K_{g} / K_{p}+2 C_{t} k_{n}\right)},
$$

where $K_{g}$ and $K_{p}$ are the thermal conductivities of the carrier gas and the particle, respectively. Batcheler and Shen [41] suggested that the thermal slip coefficient $C_{s}=1.147$, the temperature jump coefficient $C_{t}=2.18$ and the momentum exchange coefficient $C_{m}=1.146$. The Knudsen number $K_{n}=\lambda / r_{p}$, defined as the ratio of the mean free path $\lambda$ of carried fluid to the radius of the spherical particle $r_{p}$, is generally used to characterize the interaction of suspended particle and carrier fluid. Messerer et al. [42] represented that the variances of $K_{t h}$ with $K_{n}$ can be divided into three regimes: the continuum regime $\left(K_{n}<<1\right)$, the transition regime $\left(K_{n} \approx 1\right)$, and the free molecular regime $\left(K_{n} \gg 1\right)$. A constant thermophoretic coefficient $K_{n} \approx 0.55$ has been concluded to be applicable for the particles in the free molecule and transition regimes. Since the calculated values obtained by the present analysis for $K_{t h}$ remain on a constant value around $K_{n} \approx 0.55282$, the particle sizes involved in the present model may reasonably be considered to lie in the free molecule regime and come closer to the transition regime with the larger particles.

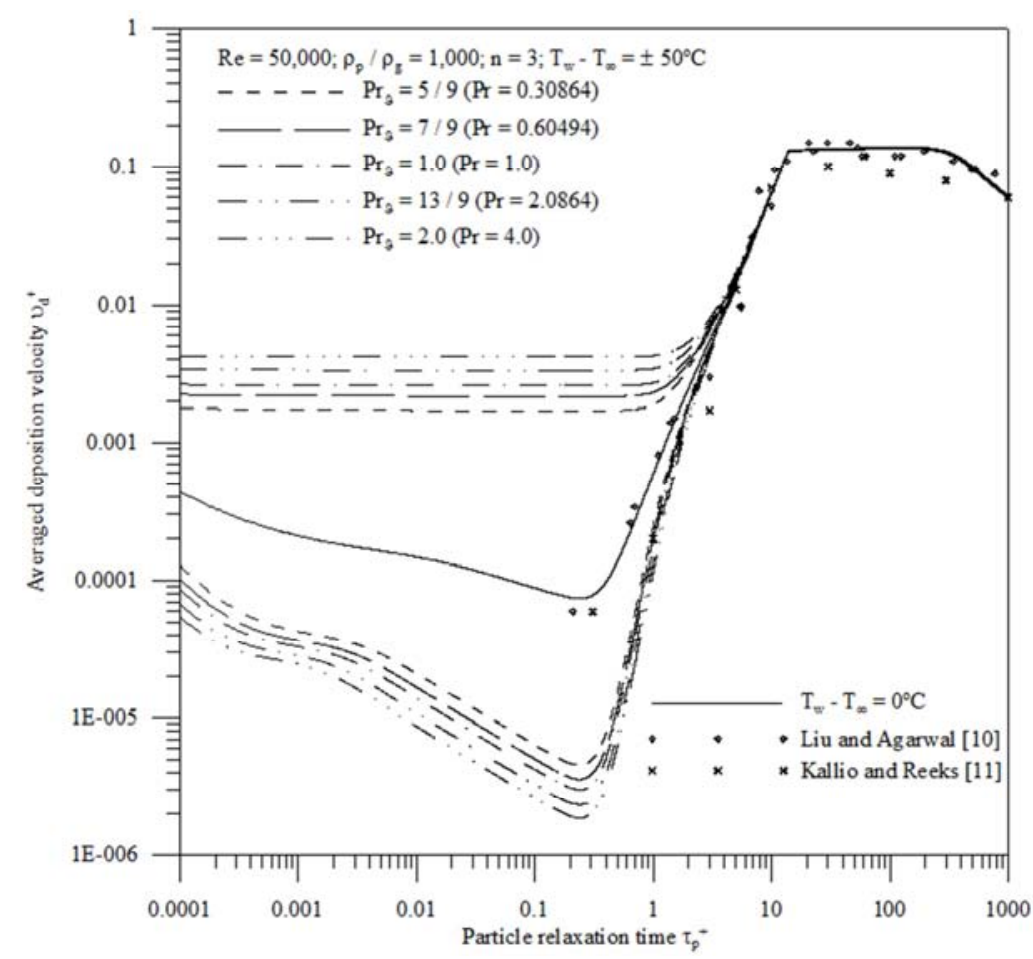

Figure 9. Changes in $\bar{v}_{d}^{+}$vs. $\tau_{p}^{+}$dependence for neutral particles provided by specifying values of transferred parameter $\vartheta\left(=\bar{H} / \sqrt{\left(\alpha+\varepsilon_{t}\right) \bar{\tau}}\right)$. 
$\operatorname{Pr}_{\vartheta}=\vartheta / \omega=\sqrt{\left(\nu+\varepsilon_{t}\right) /\left(\alpha+\varepsilon_{t}\right)} \quad$ as $\quad$ well as Prandtl number $\operatorname{Pr}=v / \alpha$ acts as an important parameter for characterizing the relative importance of momentum and energy transport by the turbulent diffusivity. For any choice of $\operatorname{Pr}_{\vartheta}$, the variations of $\bar{v}_{d}^{+}$with $\tau_{p}^{+}$in the presence of temperature difference $T_{w}-T_{\infty}= \pm 50^{\circ} \mathrm{C}$ between the wall and the bulk fluid are depicted in figure 9 for neutral particles. It is observed that the effect of changing thermal boundary layer is most pronounced in the ranges of small particle size, and the deviations from $\bar{v}_{d}^{+}$curve obtained under isothermal condition can always be increased as $\operatorname{Pr}_{\vartheta}$ is increased. Because the profile of thermal drift velocity becomes sharper and closer to the wall with respect to deeper penetrated thermal wave at the high values of $\operatorname{Pr}_{\vartheta}$, hence, the deposition flux of particles within a very narrow region close to the wall is seriously be affected by the near-wall thermal intensity gradient when the fluid turbulence level is very low. On the other hand, at low $\operatorname{Pr}_{\vartheta}$, the thermal transfer due to turbulence may be limited by the viscous drag resistance, which will consequently lead to the heat flux reduction by preventing the fluid flow from taking more heat. Figure 10 demonstrates that the enhanced heat flux gradient inside the viscous sublayer can play an important role in particle transport when an external electric filed is imposed. As can be observed, at the same value of $\operatorname{Pr}_{\vartheta}$, the significant deviations from $\bar{v}_{d}^{+}$ curve obtained under isothermal condition occurs approximately around $\tau_{p}^{+} \approx 0.3$, and the amount of this discrepancy is about the same as those of neutral particles. This emphasizes the fact that the increase in the inertial drift mechanism due to the gradient of turbulent fluctuating intensity becomes comparable to the effect of thermal intensity gradient and, eventually dominant in helping the particles of high inertia to coast across the viscous sublayer without being influenced significantly by other drift mechanisms. It is also observed that, especially in the range $\tau_{p}^{+}<0.1, \bar{v}_{d}^{+}$curve of particles with saturation charge rises more steeply than that of neutral particles, demonstrating that the gradient of heat flux adjacent to the wall can exert a profound effect on $\bar{v}_{d}^{+}$through the interaction with the Coulombic forces. However, the simulated $\bar{v}_{d}^{+}$curve is found to become identical with that solved for isothermal flow in the submicrometer-size range. This means that for submicrometer-sized and smaller particles, the gradient in the near-wall heat flux is not a major factor in the deposition mechanism. Alternatively, the Coulombic force becomes dominant in making those particles to move faster than the containing fluid and enhancing particle deposition onto the wall.

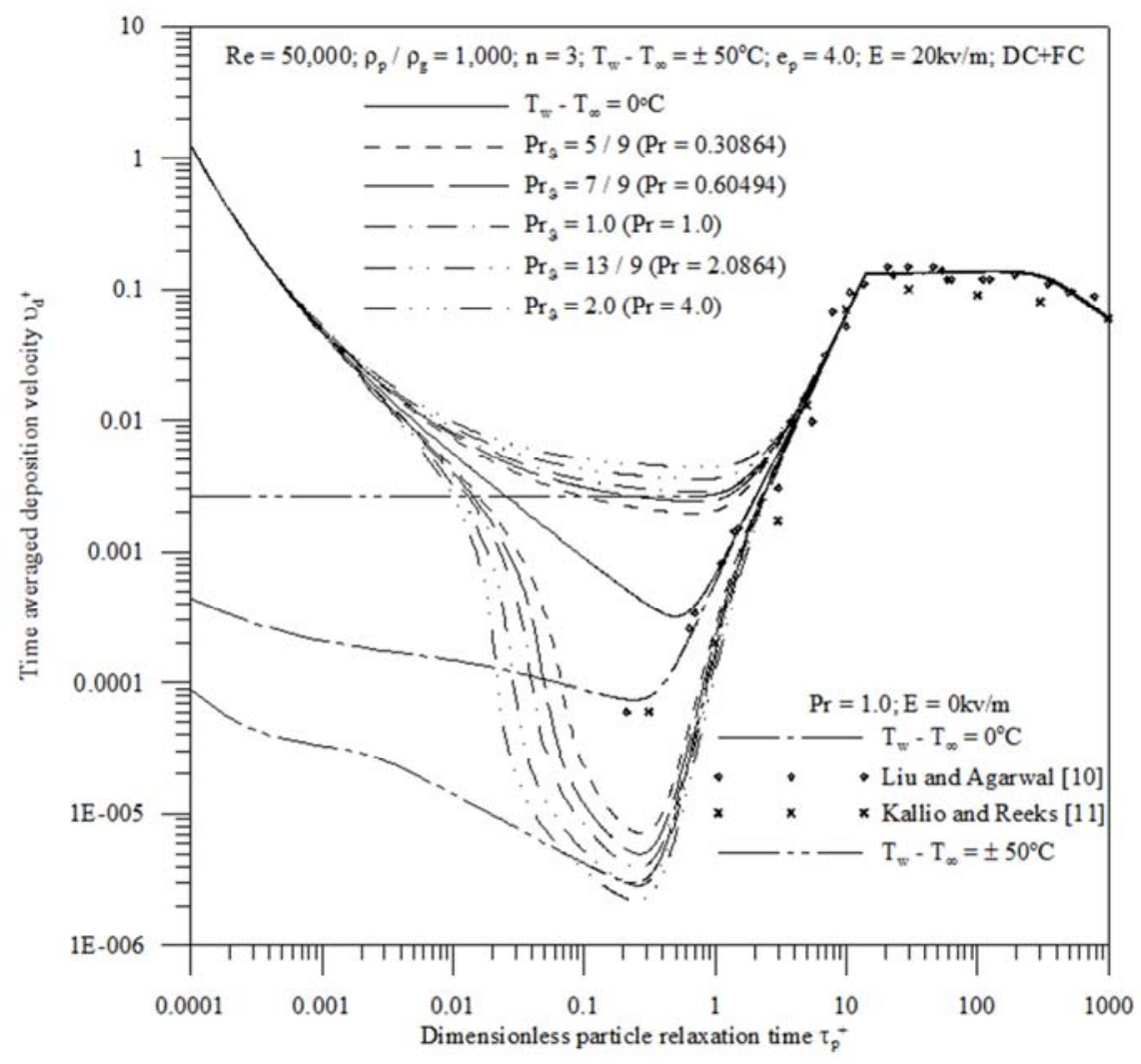

Figure 10. Changes in $\bar{v}_{d}^{+}$vs. $\tau_{p}^{+}$dependence for particles at saturation charge distribution provided by specifying values of transferred parameter $\vartheta\left(=\bar{H} / \sqrt{\left(\alpha+\varepsilon_{t}\right) \bar{\tau}}\right)$. 
Figure 11 further shows that, when the surface is heated or cooled, the temperature gradients generate thermophoretic forces on particles, which can be very significant depending on the charging mechanism, noting $\bar{v}_{d}^{+}$curves for particles with field charge supporting the existence of field charging region. As can be noted, for each set of $\bar{v}_{d}^{+}$curves, the thermophoresis induces an additional drift velocity against the near-wall thermal intensity gradient, which in turn results in a significant deviation from $\bar{v}_{d}^{+}$curve calculated under isothermal flow condition. In particular, the inclusion of thermophoresis acts not only as a complementary mechanism to enhance the particle deposit on the cooled surface, but also as a successive abatement to alleviate the particle deposition in the case of heated surface. However, there certainly exists a significant difference between $\bar{v}_{d}^{+}$curves if one includes the effect of filed charging, and the effect of thermophoresis is most marked in the field charging region.

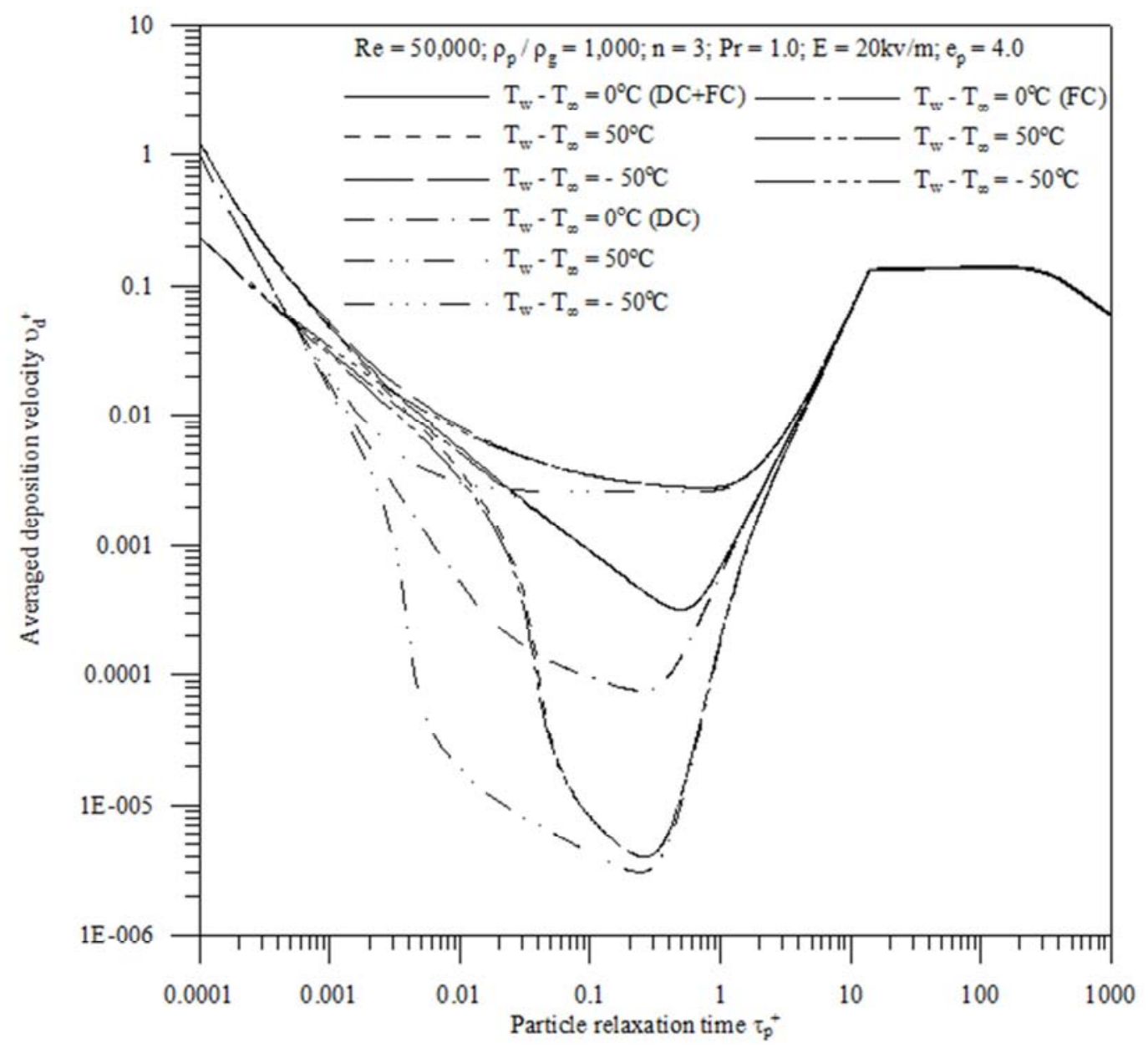

Figure 11. Effects of diffusion charging $(D F)$, field charging $(F C)$, and saturation charge distribution $(D C+F C)$ on thermophoretic behaviour of particles in the presence of an imposed electric field.

Figure 12 reproduces this characteristic dependence of $\bar{v}_{d}^{+}$ on thermophoresis for various thermal intensity gradients. The results for neutral particles are also shown in this figure for comparison. For particles that carry the saturation charge in the presence of an electric field intensity $E=20 \mathrm{kv} / \mathrm{m}$, the amount that deviated from the values of $\bar{v}_{d}^{+}$obtained under an isothermal turbulence flow condition increases with increased thermal intensity gradient adjacent to the wall. A comparison with figure 11 clearly shows that the coupling between thermophoretic and Coulombic force interactions is especially prominent for particles in the field charging region, vanishing for much smaller particles. And it is also found that, with the increase of $\tau_{p}^{+}$in the particle inertia-moderated regime, the inertial deposition mechanism induced by the gradient in turbulent fluctuating velocity adjacent to the wall becomes gradually important and eventually dominates the other deposition mechanism beyond certain $\tau_{p}^{+}$range. In consequence, these comparisons draw a very important conclusion that, when both the Coulombic and thermophoretic forces operate together, the noted discrepancy in the field charging region arises due primarily to the coupling between thermophoretic and Coulombic force interactions, but the total is not the sum of these drift mechanisms considered in isolation. 


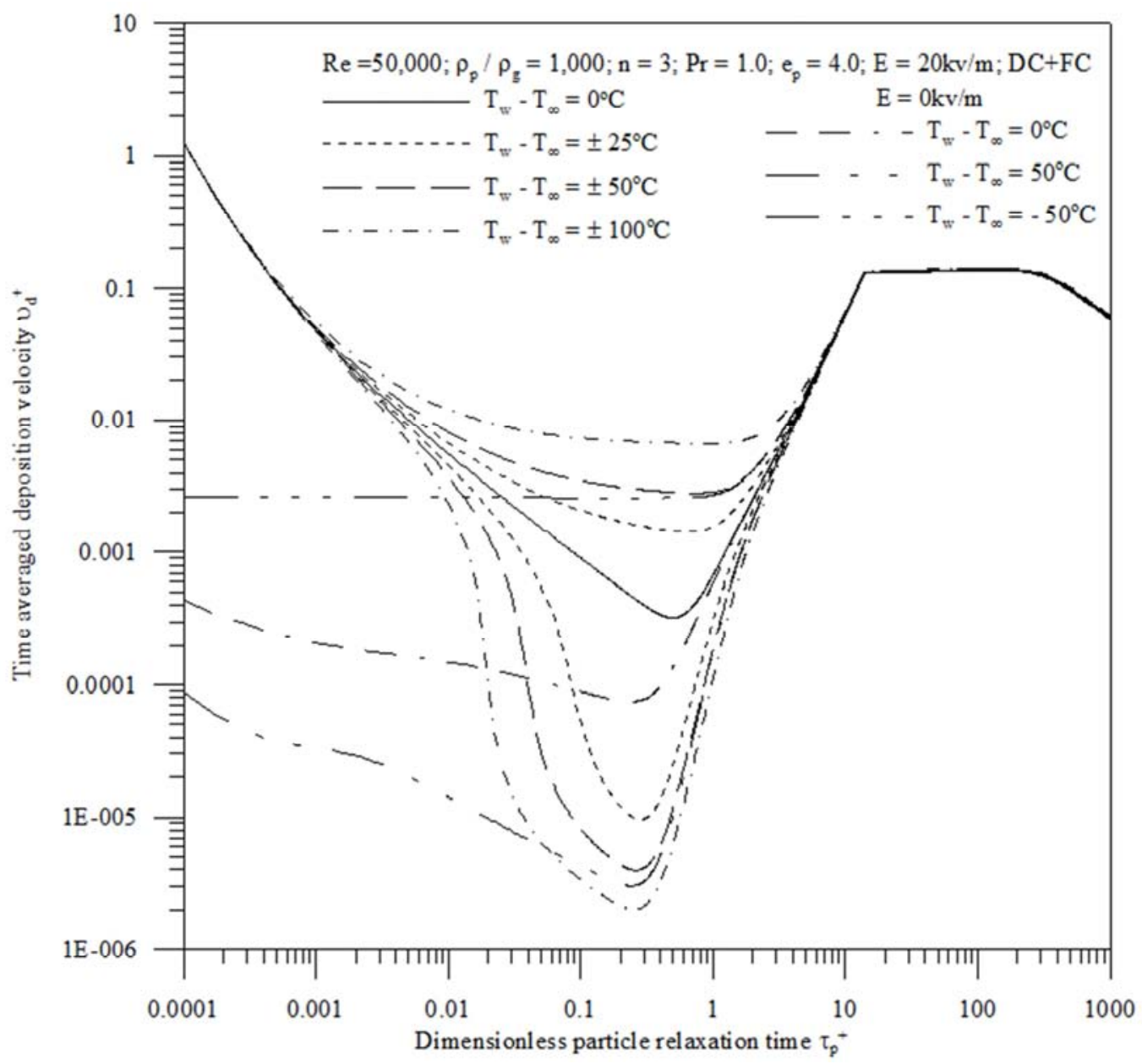

Figure 12. Combined effect of thermophoretic and Coulombic forces on $\bar{v}_{d}^{+}$vs. $\tau_{p}^{+}$dependence for particles at saturation charge distribution under various temperature differences.

The variations of $\bar{v}_{d}^{+}$for particles at the saturation charge distribution under various electric field strengths in a nonisothermal turbulent flow are plotted in figure 13 together with those for neutral particles. Especially, for particles in the field charging region, a similarity indeed exists between the thermophoretic behaviors of charged and neutral particles. Also, since the effect of applying thermophoretic force to particles is found to be most marked in the absence of external electric field, the magnitude of $\bar{v}_{d}^{+}$changes is almost identical to that of neutral particles when the intensity of imposed electric filed is dropped in this $\tau_{p}^{+}$range. On the other hand, for particles in the submicrometer-size range, there is the coincidence between $\bar{v}_{d}^{+}$curves calculated with and without thermophoretic force included, and as a result the particles can be deposited on the wall readily and thermophoresis is not a major factor in maintaining high values of $\bar{v}_{d}^{+}$. The results calculated without thermophoretic effect are also shown in this figure, which is useful in stressing the importance of the electrical field strength on $\bar{v}_{d}^{+}$and confirming the relative independence of $\bar{v}_{d}^{+}$upon the
Coulombic force effect at larger values of $\tau_{p}^{+}$. In particular, the Coulombic forces induce a wallward drift velocity to accelerate particles toward the wall despite the reduction of $\varepsilon_{p} / v$ in the turbulent diffusion-eddy impaction regime.

This leads to a wide stretch of $\bar{v}_{d}^{+}$curves, which spreads especially in the ranges of small particle size due primarily to a substantial increment of $\bar{v}_{d}^{+}$with electric field strength. It is evident that, although the thermophoretic behaviour of particles does not depend explicitly on this increment, however, the values of $\bar{v}_{d}^{+}$exhibit a tendency to be increased when the intensity of imposed electric filed is increased. As can be inferred from this increase tendency, in the presence of a relatively high electric field strength, the thermophoresis can also generate a complementary mechanism to enhance the charged particle deposit on the cooled surface, but its opposite effect on effectively alleviating the particle deposition may be abated considerably by the Coulombic force in the case of heated surface. This also demonstrates that the sum of thermophoretic and Coulombic force contributions considered separately does not represent the total deposition flux. 


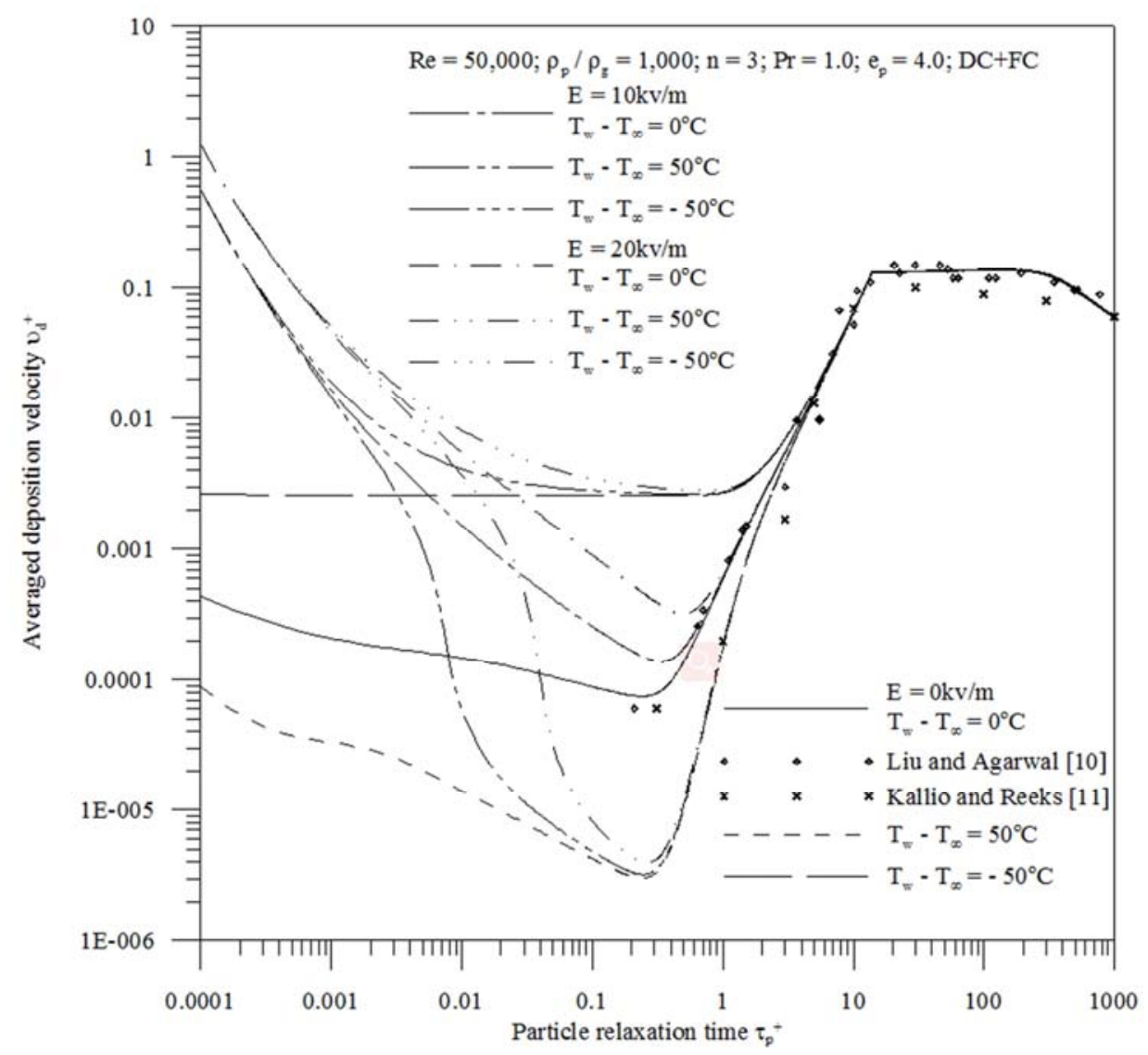

Figure 13. Combined effect of thermophoretic and Coulombic forces on $\bar{v}_{d}^{+} \quad v s . \quad \tau_{p}^{+}$dependence for particles at saturation charge distribution under various strengths of imposed electric field.

\section{Conclusions}

The proposed relationship for $\bar{v}_{d}^{+}$has been obtained by adaptation of the surface rejuvenation model to simultaneously solve both the particle continuity and momentum equations, with the inclusion the effect of Coulombic force in addition to the Brownian and turbulent diffusion, the eddy impaction, the turbophoresis, and the thermophoresis. The key to this formulation approach is that the exponentially distributed functions are introduced into the differential formulation, which transforms the differential equations and boundary conditions into the mean domain and leads to analytical solutions for the mean concentration profile and mass transfer flux. In the absence of thermophoretic and Coulombic forces, an analytical evaluation of the change in $\bar{v}_{d}^{+}$with $\tau_{p}^{+}$has been found to be in good agreement with the available experimental and numerical data. With the consideration of the coupling between thermophoretic and Coulombic force interactions in the vicinity of the wall, the thermophoresis generates a complementary mechanism to enhance the charged particle deposit on the cooled surface, but its opposite effect on effectively alleviating the particle deposition is abated considerably by the Coulombic force in the case of heated surface. This trend in $\bar{v}_{d}^{+}$is a graphic illustration of the fact that, when both the thermophoretic and
Coulombic forces operate together, the total effect on the time averaged particle flux is not the sum of these drift mechanisms considered in isolation.

\section{References}

[1] Guha, A.: A unified Eulerian theory of turbulent deposition to smooth and rough surfaces, J. Aerosol Sci. 28(8), 1517-1537 (1997).

[2] Young, J. B., Leeming, A. D.: A theory of particle deposition in turbulent pipe flow, Journal of Fluid Mechanics 340, 129-159 (1997).

[3] Danckwerts, P. V.: Significance of liquid-film coefficients in gas absorption, Ind. Eng. Chem. 43, 1460-1467 (1951).

[4] Montgomery, T. L., Corn, M.: Aerosol deposition in a pipe with turbulent airflow, J. Aerosol Sci. 1, 185-213 (1970).

[5] Friedlander, S. K., Johnstone, H. F.: Deposition of suspended particles from turbulent gas streams, Ind. Eng. Chem. 49(7), 1151-1156 (1957).

[6] Davies, C. N.: Deposition of aerosol from turbulent flow through pipes, Proc. Roy. Soc. A. 289, 235-246 (1966).

[7] Beal, S. K.: Deposition of particles in turbulent flow on channel or pipe walls, Nucl. Sci. Eng. 40, 1-11 (1970). 
[8] Liu, B. Y. H., Ilori, T. Y.: Aerosol deposition in turbulent pipe Flow, Environ Sci. Technol. 8, 351-356 (1974).

[9] Hinds, W. C.: Aerosol technology - properties, behavior, and measurement of airborne particles, vol. 2, John Wiley \& Sons. Inc. (1999).

[10] Liu, B. Y. H., Agarwal, J. K.: Experimental observation of aerosol deposition in turbulent flow, J Aerosol Sci. 5, 145-155 (1974).

[11] Kallio, G. A., Reeks, M. W.: A numerical simulation of particle deposition in turbulent boundary layers, Int. J. Multiphase Flow $15,433-446$ (1989).

[12] Popovich, A. T., Hummel, R. L.: Experimental study of the viscous sublayer in turbulent pipe flow, AIChE J. 13, 854-860 (1967).

[13] Corino, E. R., Brodkey, R. S.: A visual investigation of the wall region on turbulent flow, J. Fluid Mech. 37, 1-30 (1969).

[14] Harriott, P.: A random eddy modification of the penetration theory, Chem. Eng. Sci. 17, 149-154 (1962).

[15] Bullin, J. A., Dukler, A. E.: Random eddy models for surface renewal: formulation as a stochastic process, Chem. Eng. Sci. 27, 439-442 (1972).

[16] Brocker, S.: A new viscous sublayer influx (VSI) concept for near-wall turbulent momentum, heat and mass transfer, Rev. Gen. Therm. 37, 353-370 (1998).

[17] Meek, R. L., Baer, A. D.: The periodic viscous sublayer in turbulent flow, AIChE J. 16, 841-848 (1970).

[18] Fortuin, J. M. H., Musschenga, E. E., Hamersma, P. J.: Transfer processes in turbulent pipe flow described by the ERSR model, AIChE J. 38, 343-362 (1992).

[19] Musschenga, E. E., Hamersma, P. J., Fortuin, J. M. H.: Momentum, heat and mass transfer in turbulent pipe flow: the extended random surface renewal model, Chem. Eng. Sci. 47, 4373-4392 (1992).

[20] Mito, Y., Hanratty, T. J.: A stochastic description of wall sources in a turbulent field, Part 3: Effect of gravitational settling on the concentration profiles, Int. J. Multiphase Flow $31,155-178$ (2005).

[21] Shin, M., Kim, D. S., Lee, J. W.: Deposition of inertia-dominated particles inside a turbulent boundary layer, Int. J. Multiphase Flow 26, 892-926 (2003).

[22] Sookne, D. J.: Bessel functions of real argument and integer order, Natl. Bureau Stand. J. Res. B 77A, 125-132 (1973).

[23] He, C., Ahmadi, G.: Particle deposition in a nearly developed turbulent duct flow with electrophoresis, J. Aerosol Sci. 30, 739-758 (1999).

[24] Turner, J. R., Fissan, H. J.: Convective diffusion of particles in external force fields: The role of electrostatics on particle removal from turbulently-mixed gases, Chem. Eng. Sci. 44, 1255-1261 (1989).

[25] Cooper, D. W., Peters, M. H., Miller, R. J.: Predicted deposition of submicron particles due to diffusion and electrostatics in viscous axisymmetric stagnation-point flow, Aerosol Sci. Technol. 11, 133-143 (1989).
[26] Fan, F. G., Ahmadi, G.: On the sublayer model for turbulent deposition of aerosol particles in the presence of gravity and electric field, J. Aerosol Sci. 21, 49-71 (1994).

[27] Li, A., Ahmadi, G.: Aerosol particle deposition with electrostatic attraction in turbulent channel flow, J. Colloid Interface Sci. 158, 476-482 (1993).

[28] Soltani, M, Ahmadi, G., Ounis, H., McLaughlin, J. B.: Charged particle trajectory statistics and deposition in a turbulent channel flow, Int. J. Multiphase Flow 24, 77-92 (1998).

[29] Hartmann, G. C., Marks, L. M., Yang, C. C.: Physical models for photoactive pigment electrophotography, J. Appl. Phys. 47, 5409-5420 (1976).

[30] Hidy, G. M.: Aerosls, An Industrial and Environmental Science, Academic Press, New York (1984).

[31] Turner, J. R., Liguras, D. K., Fissan, H. J.: Clean room applications of particle deposition form stagnation flow: Electrostatic effects, J. Aerosol Science 20, 403-417 (1989).

[32] Soltani, M., Ahmadi, G.: Charged particle trajectory statistics and deposition in a turbulent channel flow, Aerosol Science and Technology 31, 170-186 (1999).

[33] Whitby, K. T., Liu, B. Y. H.: Aerosol science (edited by Davies C. N.), Academic Press, London and New York (1966).

[34] Eck, B.: Technische Stromungslehre, Springer, New York (1973).

[35] Sehmel, G. A.: Particle diffusivities and deposition velocities over a horizontal smooth surface, J. Coll. Interf. Sci. 37(4), 891-906 (1971).

[36] Flagan, R. C., Seinfeld, J. H.: Fundamentals of Air Pollution Engineering, Prentice-Hall, Englewood Cliffs, NJ, (1988).

[37] Wang, A., Song, Q., Ji, B. Q., Yao, Q., 2015. Thermophoretic motion behavior of submicron particles in boundary-layer-separation flow around a droplet. Phys. Rev. E 92, 063031 .

[38] Eslamian, M., Ahmed, M., El-Dosoky, M. E., Saghir, M. Z.: Effect of thermophoresis on natural convection in a RayleighBenard cell filled with a nanofluid, Int. J. Heat Mass Transfer 81, 142-156 (2015).

[39] Yi Zhou, Chun Yang, Yee Cheong Lam, Xiaoyang Huang, Thermophoresis of charged colloidal particles in aqueous media - Effect of particle size, Int. J. Heat Mass Transfer 101, 1283-1291 (2016).

[40] Talbot, L., Cheng, R. K., Schefer, R. W., Willis, D. R.: Thermophoresis of Particles in a Heated Boundary Layer, J. Fluid Mech. 101, 737-758 (1980).

[41] Batcheler, G. K., Shen, C.: Thermophoretic deposition of particles in gas flowing over cold surfaces, J. Colloid and Interface Sci. 107, 21-37 (1985).

[42] Messerer, A., Niessner, R., Poschl, U.: Thermophoretic deposition of soot aerosol particles under experimental conditions relevant for modern diesel engine exhaust gas systems, J. Aerosol Sci. 34, 1009-1021 (2003). 